\title{
Carbon-enhanced metal-poor stars: the most pristine objects? ${ }^{\star} \star \star \star$
}

\author{
M. Spite ${ }^{1}$, E. Caffau ${ }^{2,1}$, P. Bonifacio ${ }^{1}$, F. Spite ${ }^{1}$, H.-G. Ludwig ${ }^{2,1}$, B. Plez ${ }^{3}$, and N. Christlieb ${ }^{2}$ \\ ${ }^{1}$ GEPI Observatoire de Paris, CNRS, Université Paris Diderot, 92195 Meudon Cedex, France \\ e-mail: monique.spite@obspm. fr \\ 2 Zentrum für Astronomie der Universität Heidelberg, Landessternwarte, Königstuhl 12, 69117 Heidelberg, Germany \\ 3 LUPM, CNRS, UMR 5299, Université de Montpellier II, 34095 Montpellier Cedex 05, France
}

Received 21 December 2012 / Accepted 18 Ferbuary 2013

\begin{abstract}
Context. Carbon-enhanced metal-poor stars (CEMP) form a significant proportion of the metal-poor stars, their origin is not well understood, and this carbon-enhancement appears in stars that exhibit different abundance patterns.

Aims. Three very metal-poor C-rich turnoff stars were selected from the SDSS survey, observed with the ESO VLT (UVES) to precisely determine the element abundances. In turnoff stars (unlike giants) the carbon abundance has not been affected by mixing with deep layers and is therefore easier to interpret.

Methods. The analysis was performed with one dimensional (1D) local thermodynamical equilibrium (LTE) static model atmospheres. When available, non-LTE corrections were applied to the classical LTE abundances. The 3D effects on the CH and CN molecular bands were computed using hydrodynamical simulations of the stellar atmosphere (CO $\left.{ }^{5} B O L D\right)$ and are found to be very important. Results. To facilitate a comparison with previous results, only 1D abundances are used in the discussion. The abundances (or upper limits) of the elements enable us to place these stars in different CEMP classes. The carbon abundances confirm the existence of a plateau at $A(\mathrm{C})=8.25$ for $[\mathrm{Fe} / \mathrm{H}] \geq-3.4$. The most metal-poor stars $([\mathrm{Fe} / \mathrm{H}]<-3.4)$ have significantly lower carbon abundances, suggesting a lower plateau at $A(\mathrm{C}) \approx 6.5$. Detailed analyses of a larger sample of very low metallicity carbon-rich stars are required to confirm (or refute) this possible second plateau and specify the behavior of the CEMP stars at very low metallicity.
\end{abstract}

Key words. stars: abundances - stars: carbon - stars: AGB and post-AGB - stars: Population II - Galaxy: evolution

\section{Introduction}

Carbon-enhanced extremely metal-poor stars (CEMP) have not yet been explained satisfactorily and obviously deserve more detailed investigations. At very low metallicity many stars are carbon enriched e.g.: Rossi et al. (1999), Marsteller et al. (2005), Beers \& Christlieb (2005), Frebel et al. (2006), Lucatello et al. (2006). According to Lucatello et al., more than $20 \%$ of the metal-poor stars with $[\mathrm{Fe} / \mathrm{H}]<-2.0$ exhibit $[\mathrm{C} / \mathrm{Fe}]>+1.0$, and it has been shown (Frebel et al. 2006) that the fraction of CEMP stars increases with the distance to the Galactic plane.

Moreover, the fraction of CEMP stars seems to increase when the metallicity decreases, and a significant carbon abundance has been considered an important factor to help the condensation of clouds into stars. Of the four stars known with $[\mathrm{Fe} / \mathrm{H}]<-4.5$, only one is known with a normal abundance pattern (no enhancement of C: SDSS J02915+172927 recently discovered by Caffau et al. (2011a, 2012)), the other three are CEMP stars: the CEMP fraction is very large, but the dataset (only four stars) is so limited that according to Yong et al. (2013a) this is not statistically significant.

The carbon-enhanced stars exhibit various heavy-element abundance patterns: some are enriched in heavy elements built by both the "s" and the " $r$ " processes (CEMP-rs stars), some are enriched in only heavy elements built by the s-process (CEMP-s

\footnotetext{
* Based on observations obtained with the ESO Very Large Telescope at Paranal Observatory, Chile (ID 087.D-0123(A).

$\star \star$ Table 5 is available in electronic form at http://www. aanda.org
}

stars), some others have a normal pattern of the heavy elements (CEMP-no stars). Masseron et al. (2010) have shown that generally CEMP-s and CEMP-rs stars have abundance patterns that suggest mass-transfer from a companion in the asymptotic giant branch (AGB). A large percentage of the CEMP-s or -rs stars indeed shows radial velocity variations: this observed percentage is so large that it suggests (Lucatello et al. 2005) that all these stars are binaries. The AGB companions have different masses and abundances.

However, the abundance pattern of the CEMP-no stars is difficult to explain as caused by a mass transfer from an AGB companion. For example, HE 1327-2326 (Frebel et al. 2005, 2006; Aoki et al. 2006) is an extremely iron-poor star $([\mathrm{Fe} / \mathrm{H}]=-5.5)$ and has a ratio $[\mathrm{Sr} / \mathrm{Ba}]>-0.4$. This ratio does not seem to be compatible with a production of $\mathrm{Sr}$ and $\mathrm{Ba}$ in an AGB companion. Moreover, so far only CS 22957-027 exhibits direct evidence for duplicity (Preston \& Sneden 2001). Aoki et al. (2006) have suggested that $\mathrm{Sr}$ might have originated from the r-process $([\mathrm{Sr} / \mathrm{Ba}]=-0.4$ is the limit compatible with the classical " $\mathrm{r}$ " process) or from a "weak $r$ process" which is supposed to explain the high $[\mathrm{Sr} / \mathrm{Ba}]$ in some EMP stars with $[\mathrm{Fe} / \mathrm{H}]<-3.0$. In addition, Masseron et al. (2010) have found that the CEMP-no stars are more abundant at low metallicity and that the carbon enhancement in these stars is declining with metallicity, a trend not predicted by any of the current AGB models.

Very few CEMP stars with $[\mathrm{Fe} / \mathrm{H}]<-3.0$ have been studied, therefore observing a few CEMP stars with $[\mathrm{Fe} / \mathrm{H}] \leq-3.0$ will help to specify the behavior of the CEMP stars at very low metallicity, and shed some light on the formation of the first stars. 
Table 1. Photometric data and preliminary estimates of $[\mathrm{Fe} / \mathrm{H}]$ from SDSS spectra.

\begin{tabular}{lcccccc}
\hline \hline Star & $g$ & $g-z$ & $(g-z)_{0}$ & $\begin{array}{c}T_{\text {eff }} \\
\text { SDSS }\end{array}$ & $\begin{array}{c}T_{\text {eff }} \\
(g-z) \\
\text { adopted }\end{array}$ \\
\hline SDSS & & & & & & \\
J1114+1828 & 16.48 & 0.414 & 0.365 & -3.5 & 6300 & 6200 \\
J1143+2020 & 16.87 & 0.385 & 0.339 & -3.5 & 6300 & 6240 \\
J2209-0028 & 18.34 & 0.457 & 0.201 & -4.5 & 6400 & 6440 \\
\hline
\end{tabular}

Table 2. g magnitude of the stars and signal-to-noise ratio of the mean spectrum at different wavelengths.

\begin{tabular}{lcccc}
\hline \hline & $S / N$ & $S / N$ & $S / N$ & $S / N$ \\
Star & $395 \mathrm{~nm}$ & $445 \mathrm{~nm}$ & $588 \mathrm{~nm}$ & $777 \mathrm{~nm}$ \\
\hline SDSS & & & & \\
$\mathrm{J} 1114+1828$ & 50 & 70 & 60 & 100 \\
$\mathrm{~J} 1143+2020$ & 40 & 60 & 60 & 100 \\
$\mathrm{~J} 2209-0028$ & 17 & 30 & 30 & 48 \\
\hline
\end{tabular}

\section{Observations of the star sample}

The ratios $[\mathrm{Fe} / \mathrm{H}]$ and $[\mathrm{C} / \mathrm{Fe}]$ have been estimated for almost 30000 turnoff stars (Caffau et al. 2011b), from SDSS spectra (York et al. 2000; Abazajian et al. 2009). The resolving power of these spectra is $R \approx 2000$. Three promising (C-rich) candidates with a value of $[\mathrm{Fe} / \mathrm{H}]$ estimated to be below or equal to -3.5 , have were selected (Table 1). At present, the selection code (see Caffau et al. 2011b) selects only metal-poor turnoff stars (not giants); it is indeed more appropriate to analyze the $\mathrm{C}, \mathrm{N}$ and $\mathrm{O}$ abundances in stars before they have undergone the first dredge-up which alters the $\mathrm{C}, \mathrm{N}$ and $\mathrm{O}$ surface abundances by mixing with material processed in the star itself.

The spectra of the selected stars were then obtained at the VLT telescope with the UVES spectrograph (Dekker et al. 2000) in the course of two ESO periods. We used a 1.5"-wide slit and $2 \times 2$ on-chip binning. The resolving power (measured in the spectra) is $R \approx 39000$ with 2.8 pixels per resolution element. The signal-to-noise ratio $(\mathrm{S} / \mathrm{N})$ measured on the mean spectra (sum of the elementary spectra) is given in Table 2. The spectra cover the ranges $376<\lambda<500 \mathrm{~nm}$ (blue arm), $570<\lambda<$ $750 \mathrm{~nm}$, and $767<\lambda<947 \mathrm{~nm}$ (red arm).

\section{Radial velocity measurements}

Since CEMP stars are suspected to often be binary stars, it is important to measure the radial velocity of the stars as precisely as possible at the time of the observation (Table 3 ). These radial velocities were measured on the blue spectra and the estimated error is about $1.0 \mathrm{~km} \mathrm{~s}^{-1}$. The barycentric radial velocities are given in Table 3. The radial velocities of SDSS J1114+1828 and SDSS J1143+2020 are significantly different from the values given in the SDSS DR9 science archive server (see Table 3), and therefore these two stars are very likely binaries. In contrast, the radial velocity of SDSS J2209-0028 does not show any variation between 2003 and 2011.

\section{Analysis}

The effective temperature was derived from the $(g-z)_{0}$ color (Table 1) using the calibration presented in Ludwig et al. (2008). The reddening correction is from Schlegel et al. (1998).
Table 3. Radial velocities in $\mathrm{km} \mathrm{s}^{-1}$ measured on the individual UVES spectra.

\begin{tabular}{|c|c|c|c|c|c|}
\hline Date & MJD & $\mathrm{RV}_{\mathrm{G}}$ & $V_{\text {cor }}$ & $\mathrm{RV}_{\mathrm{B}}$ & Err \\
\hline \multicolumn{6}{|l|}{$\mathrm{J} 1114+1828$} \\
\hline $4 / 05 / 2011$ & 55656.1673857 & 234.9 & -15.5 & 219.4 & 1. \\
\hline 24/05/2011 & 55705.9692687 & 247.9 & -28.3 & 219.6 & 1. \\
\hline $25 / 05 / 2011$ & 55706.0053931 & 247.6 & -28.4 & 219.2 & 1. \\
\hline $25 / 05 / 2011$ & 55706.0424672 & 248.0 & -28.5 & 219.5 & 1. \\
\hline 03/06/2011 & 55715.0406638 & 247.9 & -28.9 & 219.0 & 1. \\
\hline 19/12/2007* & 54453.0024860 & & & 31.3 & 4. \\
\hline \multicolumn{6}{|l|}{$\mathrm{J} 1143+2020$} \\
\hline $22 / 06 / 2011$ & 55715.0808670 & 260.1 & -28.2 & 231.9 & 1. \\
\hline $30 / 12 /$ & 55925.3 & 202.0 & 27.4 & 229.4 & 1. \\
\hline 31/12/2011 & 55926.2907973 & 202.0 & 27.2 & 229.2 & 1. \\
\hline $22 / 02 / 2012$ & 55979.2557883 & 222.0 & 7.8 & 229.8 & 1. \\
\hline $22 / 03 / 2012$ & 56008.1214818 & 234.9 & -6.3 & 228.6 & 1. \\
\hline 20/03/2007* & 54179.3732200 & & & 202.7 & 4. \\
\hline \multicolumn{6}{|l|}{ J2209-0028 } \\
\hline 3/06/2011 & & -252.4 & 29.0 & -223.4 & 1. \\
\hline 28/07/2011 & 55770.3 & -237.3 & 13.7 & -223.6 & 1. \\
\hline $3 / 08 / 2011$ & 55776.1416334 & -234.2 & 11.7 & -222.5 & 1. \\
\hline $3 / 08 / 2011$ & 55776.1780564 & -233.9 & 11.6 & -222.3 & 1. \\
\hline $3 / 08 / 2011$ & 55776.2150359 & -233.9 & 11.5 & -222.4 & 1. \\
\hline $3 / 08 / 2011$ & 55776.2520017 & -234.5 & 11.3 & -223.2 & 1. \\
\hline $3 / 08 / 2011$ & 55776.2895941 & -234.5 & 11.2 & -223.3 & 1. \\
\hline $3 / 08 / 2011$ & 55776.3270350 & -234.5 & 11.1 & -223.4 & 1. \\
\hline $5 / 08 / 2011$ & 55778.3313394 & -232.8 & 10.2 & -222.6 & 1. \\
\hline $25 / 08 / 2011$ & 55798.1409815 & -222.2 & 1.1 & -221.1 & 2. \\
\hline 20/09/2003* & 52902.2996680 & & & -222.2 & 11. \\
\hline
\end{tabular}

Notes. The date of the observation is given in the first column, the second column gives the modified Julian date (MJD), the third column is the geocentric radial velocity $\left(\mathrm{RV}_{\mathrm{G}}\right)$ at the time of the observation, the fourth column lists the barycentric correction, and the two last columns provide the barycentric radial velocity $\mathrm{RV}_{\mathrm{B}}$ and the corresponding error. An asterisk after the observation date means that the data correspond to the radial velocity measurement in the SDSS DR9 Science Archive Server.

For comparison, we also determined effective temperatures by means of fitting synthetic line profiles to the wings of the observed $\mathrm{H} \alpha$ lines. These temperatures agree well with those derived from photometry (see Table 1 ).

The gravity was fixed at $\log g=4.0$, but we checked the ionization equilibrium of iron when some Fe II lines could be measured. The microturbulent velocity was assumed to be $1.3 \mathrm{~km} \mathrm{~s}^{-1}$. In the three stars, the metallic lines are very weak and not sensitive to the adopted value. We carried out a classical 1D LTE analysis using MARCS models (e.g. Gustafsson et al. $1975,2003,2008)$. The abundance analysis was performed using the LTE spectral line analysis code turbospectrum (Alvarez \& Plez 1998; Plez 2012). The adopted solar abundances are given in Table 4: Fe is taken from Caffau et al. (2011c), the other elements are taken from Lodders et al. (2009).

The carbon abundance was determined by fitting the $\mathrm{C}$ I line at $493.205 \mathrm{~nm}$ and the $\mathrm{CH}$ band at $422.4 \mathrm{~nm}$ ( $\mathrm{G}$ band), and the nitrogen abundance by fitting the $\mathrm{CN}$ band at $388.8 \mathrm{~nm}$. The molecular data corresponding to the $\mathrm{CH}$ and $\mathrm{CN}$ bands are 
M. Spite et al.: Carbon enhanced extremely metal-poor stars
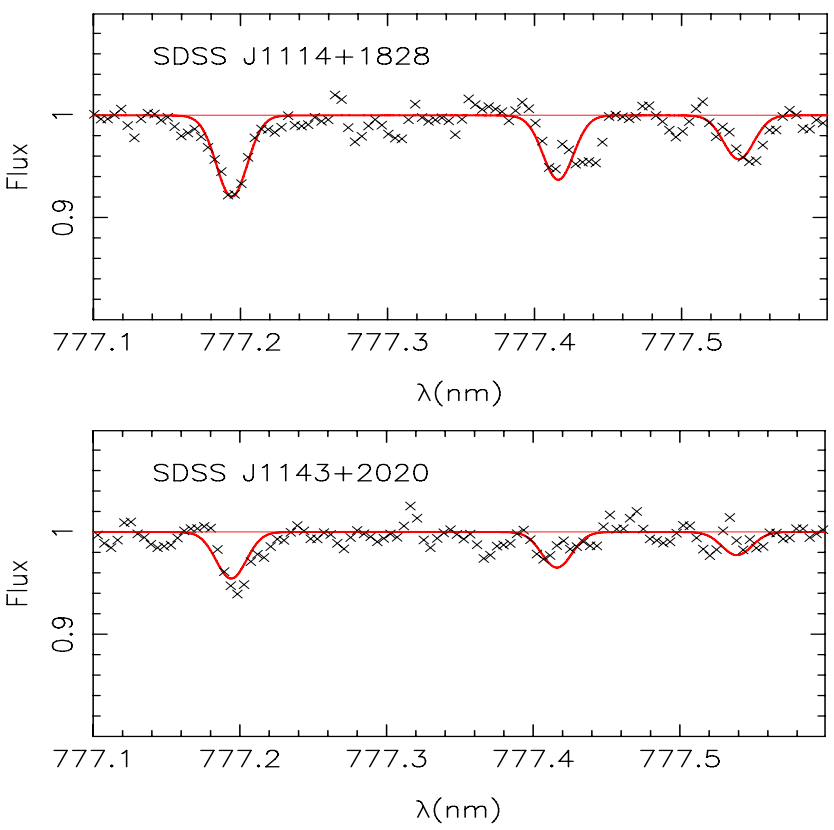

Fig. 1. UVES spectrum in the region of the red O I triplet.

described in Hill et al. (2002) and Plez et al. (2008). The oxygen abundance was deduced from the IR triplet at $777 \mathrm{~nm}$.

In Table 5 all the lines used to derive abundances are listed with their wavelengths, excitation potentials, and oscillator strengths. The word "syn" in place of the equivalent width means that the abundance was derived from spectral synthesis.

\subsection{D computations - non-LTE (NLTE) effects}

In extremely metal-poor stars such as the three stars we studied, NLTE effects are often significant: the collision rates are reduced (decrease of the electron density with metal abundance), and since the radiation field is absorbed by a smaller number of metal atoms and ions, the photoionization rate tends to increase with decreasing metal abundance (Gehren et al. 2004).

One C I line is visible in our spectra at $493.205 \mathrm{~nm}$. This high-excitation potential line is very sensitive to NLTE effects, and following Behara et al. (2010), using the Kiel code (Steenbock \& Holweger 1984) and the carbon model atom described in Stürenburg \& Holweger (1990), the correction amounts to about -0.45 dex in similar turnoff stars. This correction has been applied to the LTE value of the carbon abundance deduced from the $\mathrm{C} I$ line.

The abundance of oxygen was computed from the red permitted O I triplet (Fig. 1). NLTE corrections to oxygen abundances were computed using the Kiel code (Steenbock \& Holweger 1984), and the model atom used in Paunzen et al. (1999). We used the Steenbock \& Holweger (1984) formalism to treat the collisions with neutral hydrogen. We adopted a scaling factor $S_{\mathrm{H}}=1 / 3$, as recommended by Caffau et al. (2008); the differences between the abundancescomputed with $S_{\mathrm{H}}=0$ and $S_{\mathrm{H}}=1$ is less than 0.01 (see Behara et al. 2010).

The abundances of the light metals from sodium to calcium were corrected for NLTE effects following Andrievsky et al. (2007, 2008); Spite et al. (2012a); Mashonkina (2013).

The green magnesium triplet (multiplet 2) is beyond the wavelength range of our spectra and thus we deduced the magnesium abundance from multiplet 3 (at $383 \mathrm{~nm}$ ). Mashonkina (2013) has computed the NLTE correction for Mg taking into

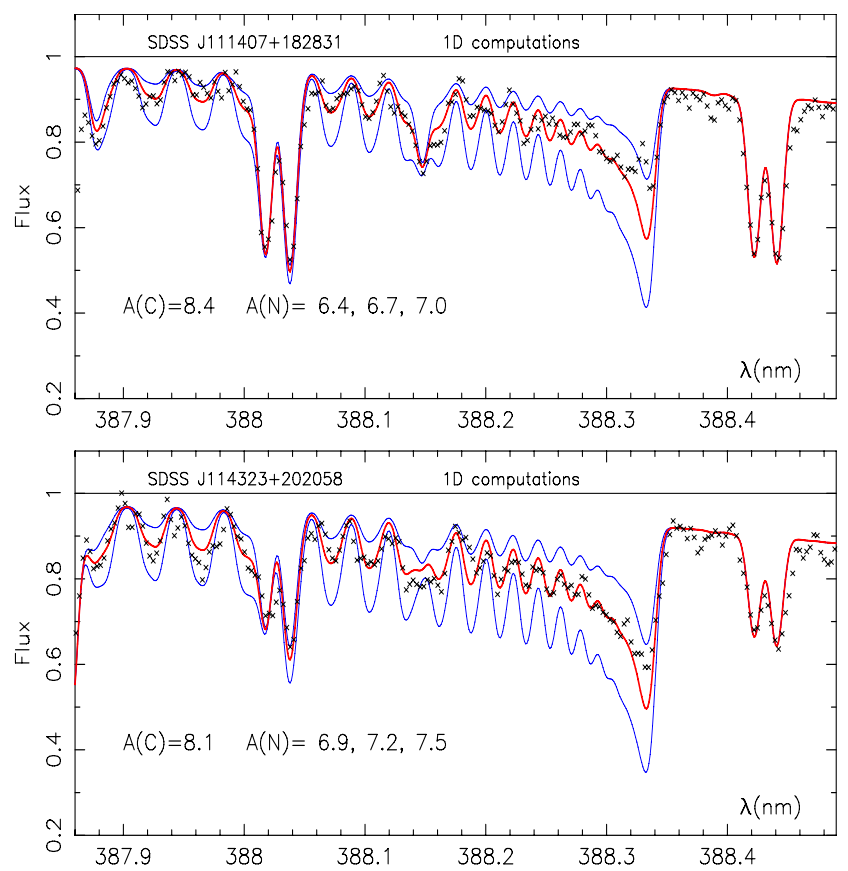

Fig. 2. UVES spectrum in the region of the $\mathrm{CN}$ band compared to theoretical spectra. The abscissa is the wavelength in $\mathrm{nm}$, and the ordinate the relative flux. For SDSSJ1114+1828 the theoretical spectra are computed with $A(\mathrm{C})=8.4$ and $A(\mathrm{~N})=6.4,6.7$ (best fit), 7.0, and for SDSSJ1143+2020, with $A(\mathrm{C})=8.1$ and $A(\mathrm{~N})=6.9,7.2$ (best fit), 7.5.

account inelastic collisions with neutral hydrogen atoms following Barklem et al. (2012). From her computations on a small grid of models, the NLTE correction for multiplet 3 amounts to about +0.14 dex for SDSSJ $1114+1828,0.10 \mathrm{dex}$ for SDSSJ $1143+2020$ and 0.25 dex for SDSSJ 2209-0028.

The manganese abundances were derived by fitting synthetic spectra of the resonance lines $(403 \mathrm{~nm})$ to observations, taking into account the hyperfine structure of the lines. Cayrel et al. (2004) underlined that the manganese abundance deduced from the resonance lines was 0.4 dex lower (in giants) than the abundance deduced from the subordinate lines of Mn I. Moreover Bonifacio et al. (2009) have shown that there is a systematic difference between the ratio $[\mathrm{Mn} / \mathrm{Fe}]$ in the turnoff stars and the giants even if only the resonance lines are taken into account. This behavior suggests a strong NLTE effect. The NLTE correction of the manganese abundance was computed for a grid of dwarf and subgiant models by Bergemann \& Gehren (2008). Their most metal-poor model of turnoff star has a metallicity of -2.4 and for this metallicity the NLTE correction amounts to about +0.5 dex. This correction was applied to our LTE abundance.

Lai et al. (2008) and Bonifacio et al. (2009) have shown an offset between the chromium abundance derived from $\mathrm{Cr}$ I and Cr II lines in metal-poor stars, that points to NLTE effects. Bergemann \& Cescutti (2010) have computed the NLTE correction for a set of $\mathrm{Cr}$ lines in metal-poor stars. The atmospheric parameters of one star (G 64-12) are close to those of our stars and thus we applied the NLTE correction found for the stronger resonance line of $\mathrm{Cr}$ I in G 64-12 (the only $\mathrm{Cr}$ line visible in our spectra): +0.44 dex.

The abundances of the heavy elements $\mathrm{Sr}$ and $\mathrm{Ba}$ are generally sensitive to NLTE effects. The abundance of $\mathrm{Sr}$ was corrected following Andrievsky et al. (2011). For Ba it has been shown (Andrievsky et al. 2009) that the NLTE correction depends almost only on $[\mathrm{Ba} / \mathrm{H}]$ (the NLTE correction of 
Table 4. Elemental abundances in the observed stars from a 1D analysis.

\begin{tabular}{|c|c|c|c|c|c|c|c|c|c|c|c|c|c|}
\hline \multicolumn{2}{|c|}{$\begin{array}{c}- \\
\text { Model }\end{array}$} & \multicolumn{4}{|c|}{$\begin{array}{c}\text { SDSS J111407.07+182831.7 } \\
6200,4.0,-3.3,1.3\end{array}$} & \multicolumn{4}{|c|}{$\begin{array}{c}\text { SDSS J114323.42+202058.0 } \\
6240,4.0,-3.3,1.3\end{array}$} & \multicolumn{4}{|c|}{$\begin{array}{c}\text { SDSS J220924.74-002859.8 } \\
6440,4.0,-4.0,1.3\end{array}$} \\
\hline Species & $\log \epsilon_{\odot}$ & $\log \epsilon$ & {$[\mathrm{M} / \mathrm{H}]$} & {$[\mathrm{M} / \mathrm{Fe}]$} & $N$ & $\log \epsilon$ & {$[\mathrm{M} / \mathrm{H}]$} & {$[\mathrm{M} / \mathrm{Fe}]$} & $N$ & $\log \epsilon$ & {$[\mathrm{M} / \mathrm{H}]$} & {$[\mathrm{M} / \mathrm{Fe}]$} & $N$ \\
\hline $\mathrm{C}(\mathrm{CH})$ & 8.50 & 8.40 & -0.10 & 3.25 & & 8.10 & -0.40 & 2.75 & & 7.10 & -1.40 & 2.56 & \\
\hline$* \mathrm{C}(\mathrm{C}$ I $)$ & 8.50 & 7.95 & -0.55 & 2.80 & 1 & 7.75 & -0.75 & 2.40 & 1 & - & - & - & \\
\hline $\mathrm{N}(\mathrm{CN})$ & 7.86 & 6.70 & -1.16 & 2.19 & & 7.18 & -0.68 & 2.47 & & - & - & - & \\
\hline$* \mathrm{O}$ & 8.76 & 7.31 & -1.45 & 1.90 & 3 & 7.00 & -1.76 & 1.39 & 3 & 6.97 & -1.79 & 2.17 & 3 \\
\hline$* \mathrm{Na}$ & 6.30 & 4.58 & -1.72 & 1.63 & 2 & 4.54 & -1.76 & 1.39 & 2 & $\leq 2.37$ & $\leq-3.93$ & $\leq 0.03$ & 2 \\
\hline$* \mathrm{Mg}$ & 7.54 & 5.65 & -1.89 & 1.46 & 4 & 5.13 & -2.41 & 0.74 & 4 & 4.30 & -3.24 & 0.72 & 3 \\
\hline$* \mathrm{Al}$ & 6.47 & 3.48 & -2.99 & 0.36 & 1 & 3.20 & -3.27 & -0.12 & 1 & - & - & - & \\
\hline$* \mathrm{Ca}$ & 6.33 & 3.35 & -2.98 & 0.37 & 6 & 3.49 & -2.84 & 0.31 & 6 & 2.92 & -3.41 & 0.55 & 5 \\
\hline $\mathrm{Ti}$ & 4.90 & 1.86 & -3.04 & 0.31 & 11 & 2.03 & -2.87 & 0.28 & 11 & - & - & - & \\
\hline$* \mathrm{Cr}$ & 5.64 & 2.49 & -3.15 & 0.20 & 1 & 2.69 & -2.95 & -0.20 & 1 & - & - & - & \\
\hline$* \mathrm{Mn}$ & 5.37 & 2.00 & -3.37 & -0.03 & 1 & 2.25 & -3.12 & 0.03 & 1 & - & - & - & \\
\hline $\mathrm{Fe} \mathrm{I}$ & 7.52 & 4.17 & -3.35 & 0.00 & 36 & 4.37 & -3.15 & 0.00 & 40 & 3.56 & -3.96 & 0.0 & 6 \\
\hline Fe II & 7.52 & 4.18 & -3.34 & 0.00 & 3 & 4.24 & -3.28 & -0.13 & 3 & - & - & & \\
\hline Co & 4.92 & 2.40 & -2.52 & 0.83 & 3 & 2.26 & -2.66 & 0.49 & 3 & - & - & - & \\
\hline $\mathrm{Ni}$ & 6.23 & 2.96 & -3.27 & 0.07 & 2 & 2.98 & -3.25 & -0.10 & 2 & - & - & - & \\
\hline$* \mathrm{Sr}$ & 2.92 & 0.29 & -2.63 & 0.72 & 2 & 0.50 & -2.42 & 0.73 & 2 & $<-1.2$ & $<-4.12$ & $<-0.16$ & 1 \\
\hline$* \mathrm{Ba}$ & 2.17 & 0.44 & -1.73 & 1.62 & 3 & 0.86 & -1.31 & 1.84 & 3 & $<-0.74$ & $<-2.91$ & $<+1.05$ & 1 \\
\hline $\mathrm{Eu}$ & 0.52 & $<-1.10$ & $<-1.62$ & $<1.73$ & 1 & $<-1.00$ & $<-1.52$ & $<1.63$ & 1 & - & - & - & \\
\hline$[\mathrm{C} / \mathrm{O}]$ & & & 1.35 & & & & 1.36 & & & & 0.29 & & \\
\hline$[\mathrm{Ba} / \mathrm{Eu}]$ & & & $>0.1$ & & & & $>0.4$ & & & & & & \\
\hline${ }^{12} \mathrm{C} /{ }^{13} \mathrm{C}$ & & & $>60$ & & & & $>20$ & & & & & & \\
\hline
\end{tabular}

Notes. Abundances of $\mathrm{C}(\mathrm{C} \mathrm{I}), \mathrm{O}, \mathrm{Na}, \mathrm{Mg}, \mathrm{Al}, \mathrm{Ca}, \mathrm{Sr}$, and Ba have been corrected for NLTE effects $(*)$, the LTE abundances for these elements are given line by line in Table 5.

the $\mathrm{Ba}$ lines is almost the same for two stars with different $[\mathrm{Fe} / \mathrm{H}]$ but the same $[\mathrm{Ba} / \mathrm{H}])$. Since SDSS J1114+1828 and SDSS J1143+2020 are Ba-rich, $[\mathrm{Ba} / \mathrm{H}]$ in these two stars is between -1.3 and -1.8 dex. For these values of $[\mathrm{Ba} / \mathrm{H}]$, the NLTE correction, following Mashonkina et al. (1999), is close to zero (see their Table 4).

The line-by-line LTE abundances of the elements with the NLTE correction (when available) are given in Table 5, and the resulting mean abundances are given in Table 4.

\subsection{Effects of $3 D$ hydrodynamical convection on the formation of molecular bands}

Classical models such as MARCS, have a number of simplifying assumptions: the atmosphere is approximated by a onedimensional structure; all the physical quantities vary along the vertical direction, but are constant in the horizontal direction for any given depth; the atmosphere is supposed to be in hydrostatic equilibrium; all quantities are time independent; the stellar plasma is supposed to be in LTE, i.e. the particle velocities are given by a Maxwellian distribution at a local temperature $T$, the atomic populations are given by Boltzmann factors, at the same temperature $T$, ionization equilibria are also given by Saha's law, at the same temperature. In cool stars convection is a hydrodynamical, time-dependent phenomenon, that needs to be treated in full three dimensional geometry. To treat these effects it is necessary to use hydrodynamical simulations, like those provided by the CO ${ }^{5}$ BOLD code (Freytag et al. 2012), which are fully hydrodynamical, time dependent, in a three dimensional frame, yet retaining the LTE assumption. In this investigation we concentrated on the hydrodynamical effects on molecular bands, because they have been shown to be extremely strong in metal-poor stars (Collet et al. 2007; Behara et al. 2010; González Hernández et al. 2010).
To estimate these effects we used a model extracted from the latest version of the CIFIST grid (Ludwig et al. 2009). The model has the parameters $T_{\text {eff }} / \log g /[\mathrm{M} / \mathrm{H}] 6300 / 4.0 /-3.0$, and consists of a box of $140 \times 140 \times 150$ points, corresponding to physical dimensions $26 \times 26 \times 13 \mathrm{Mm}^{3}$. The opacity was derived from the MARCS suite (Gustafsson et al. 1975, 2003, 2008) and was binned into 12 opacity bins following the usual opacity binning scheme (Nordlund 1983; Ludwig 1992). Behara et al. (2010) noted a considerable difference between models computed with 6 opacity bins and those computed with 12 opacity bins.

The line formation in the $3 \mathrm{D}$ structure was computed using Linfor3 $D^{1}$. For the spectrum synthesis we used a selection of 20 snapshots, chosen to be statistically independent and representative of the overall characteristics of the hydrodynamical simulation. For comparison, Linfor3D also integrates the transfer equation through two reference one-dimensional structures:

- an $1 D_{\text {LHD }}$ model (see Caffau \& Ludwig 2007) that uses the same microphysics as CO ${ }^{5}$ BOLD; and

- a $\langle 3 \mathrm{D}\rangle$ model, that is obtained by averaging the three dimensional structure over surfaces of constant Rosseland optical depth and over time.

Following Caffau \& Ludwig (2007), we defined the 3D correction as the difference in the abundance derived from the full $3 \mathrm{D}$ synthesis and that derived from the $1 \mathrm{D}_{\mathrm{LHD}}$ model $(3 \mathrm{D}-$ $\left.1 \mathrm{D}_{\mathrm{LHD}}\right)$. It is also useful to consider the difference between the abundance derived from the 3D model and the $\langle 3 \mathrm{D}\rangle$ model. Since the two models have by construction the same mean temperature distribution, this difference helps to single out the effect of temperature fluctuations.

\footnotetext{
1 http://www.aip.de/ mst/Linfor3D/linfor_3D_manual. pdf
} 


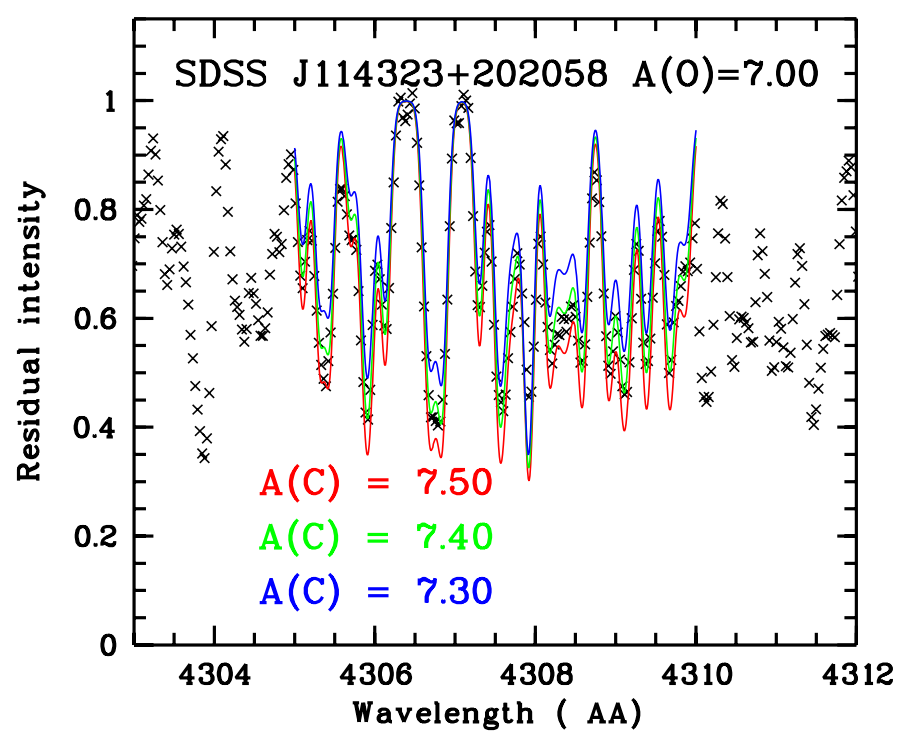

Fig. 3. UVES spectrum of SDSS J114323+202058 in the core of the $\mathrm{G}$ band (black line), compared with two Linfor3D spectral syntheses with the $\mathrm{CO}^{5} \mathrm{BOLD}$ model, corresponding to $A(\mathrm{C})=7.30,7.40$ and 7.50. The best fit is obtained with $A(C)=7.40$.

Computing molecular bands is CPU-intensive, since many lines need to be treated. The G-band is very challenging since it extends over almost $20 \mathrm{~nm}$. The data for the G-band (CH AX electronic system) were taken from Plez \& Cohen, (2005) and Plez et al. (2008). We computed the full 3D synthesis of the $G$ band core for various carbon abundances and compared them with the observed spectrum. The result for SDSS J114323+202058 is shown in Fig. 3. The best fit is obtained for $A(C)=7.4$ and as a consequence, the $1 \mathrm{D}$ to $3 \mathrm{D}$ correction is about -0.7 dex.

For the nitrogen abundance we used the $\mathrm{CN} \mathrm{BX}\left[\mathrm{B}^{2} \Sigma^{+}-\right.$ $\left.\mathrm{X}^{2} \Sigma^{+}(0-0)\right]$ violet band at $388 \mathrm{~nm}$. We used the molecular data from Hill et al. (2002) and Plez et al. (2008), but considering the limited spectral range covered by the band (about $0.5 \mathrm{~nm}$ ), it is feasible to synthetise the whole band, as shown in Fig. 4. The 3D effect seems to be much more important for this band than for the $\mathrm{CH}$ band. This band is formed very close to the surface of the star and the nitrogen abundance deduced from this band is also strongly affected by the quantity of oxygen atoms bound at this level in the $\mathrm{CO}$ molecule. As a consequence, we found for these oxygen-rich stars, a huge correction: about -2.4 dex. This correction (if confirmed) would almost annihilate the nitrogen enhancement in the CEMP turnoff stars. These 3D corrections affect not only the three turnoff stars we studied but all CEMP stars in the literature where these 3D effects have generally been neglected. These effects could be so significant that they could lead to a revision of the interpretation of the abundances in these stars (type of AGB stars responsible for the abundance anomalies). In Fig. 4 we compare the $1 \mathrm{D}$ and the $3 \mathrm{D}$ profiles of the $\mathrm{CN}$ band in SDSS J1143+2020 with different abundances.

To confirm this very large correction of the nitrogen abundance, it would be very important to compare the abundance deduced from the $\mathrm{CN}$ band and from the NH band, which is practically independent of the oxygen abundance. But the $\mathrm{NH}$ band is not in the observed spectral range of the very faint stars studied in the present paper. We will study this effect, which is very sensitive to the most external layers of the models, in another sample of CEMP stars with known $\mathrm{CN}$ and $\mathrm{NH}$ bands in a future investigation.

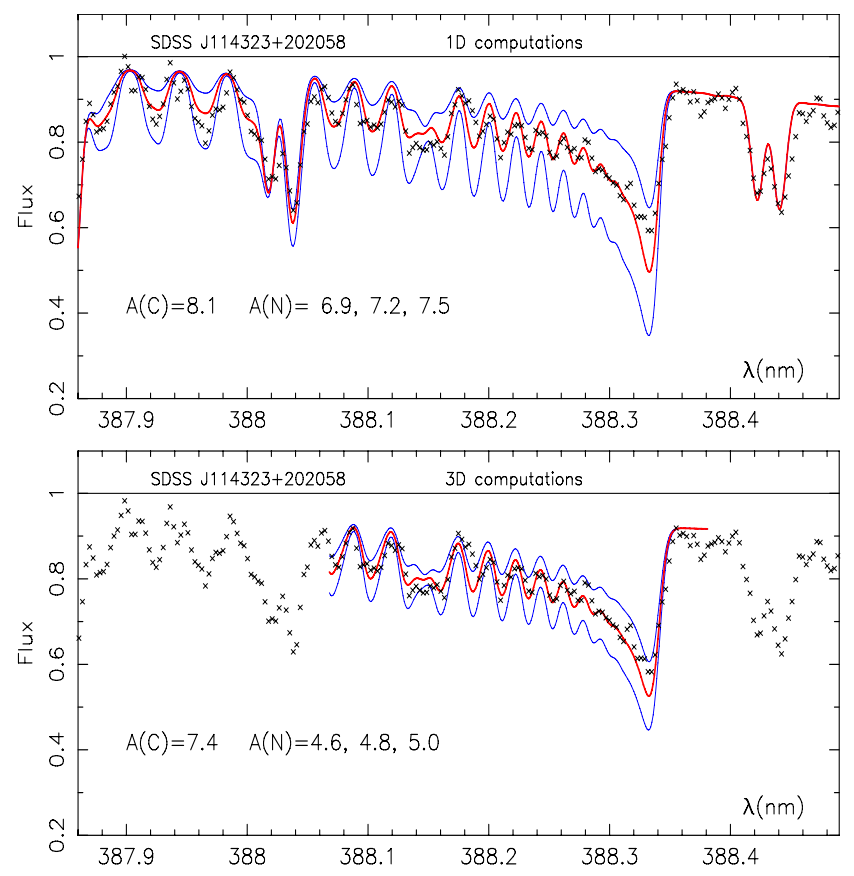

Fig. 4. UVES spectrum in the region of the $\mathrm{CN}$ band of SDSS J1143+2020. The abscissa is the wavelength in $\mathrm{nm}$, and the ordinate is the relative flux. In the top panel the observed spectrum (crosses) is compared to $1 \mathrm{D}$ theoretical spectra computed with $A(\mathrm{C})=8.1$ (best fit for the $\mathrm{CH}$ band in $1 \mathrm{D}$ ) and $A(\mathrm{~N})=6.9,7.2$ (best fit), 7.5. In the bottom panel the observed spectrum is compared to $3 \mathrm{D}$ theoretical spectra computed with $A(\mathrm{C})=7.4$ (best fit for the $\mathrm{CH}$ band in 3D, see Fig. 3) and $A(\mathrm{~N})=4.6,4.8$ (best fit) and 5.0.

Since the aim of the present paper is to enlarge the sample of the abundance patterns in CEMP stars, we discuss in the remainder of this paper the $1 \mathrm{D}$ abundances to facilitate a comparison with the previous analyses, but with the caveat that the abundance of $\mathrm{C}$ and $\mathrm{N}$ could be strongly reduced.

\subsection{SDSS J1114+1828 and SDSSJ1143+2020}

Both stars are very metal-poor $([\mathrm{Fe} / \mathrm{H}]<-3.0)$ and exhibit a very strong enhancement of $\mathrm{C}, \mathrm{N}, \mathrm{O}$ and $\mathrm{Mg}$ (Table 4). This enhancement tends to decrease with the atomic number. The $\mathrm{CN}$ feature is displayed in Fig. 2.

The carbon abundance deduced from the $\mathrm{C}$ I line is systematically lower by about 0.4 dex than the abundance deduced from the $\mathrm{CH}$ band. This difference has also been observed by Behara et al. (2010). A 3D computation of the $\mathrm{CH}$ band and the $\mathrm{C}$ I lines does not remove this discrepancy, but in this case the abundance of $\mathrm{C}$ derived from the $\mathrm{C}$ I line is higher than the abundance derived from the $\mathrm{CH}$ band. Since in the literature the carbon abundance is generally deduced from $1 \mathrm{D}$ computation of the $\mathrm{CH}$ band we used the $\mathrm{C}(\mathrm{CH})$ abundance in the forthcoming discussion.

The overabundance of $\mathrm{C}, \mathrm{N}$, and $\mathrm{O}$ in these stars is generally attributed to a production in an AGB companion that transfers some processed material to the observed star. This hypothesis is reinforced by the fact that the radial velocity of both stars have been found to be variable in Sect. 3. In Fig. 5 we plot these two new CEMP turnoff stars in a diagram $[\mathrm{N} / \mathrm{Fe}]$ vs. $[\mathrm{C} / \mathrm{Fe}]$ for comparison with the values found for other CEMP dwarfs and turnoff stars (Sivarani et al. 2006; Behara et al. 2010; Masseron et al. 2012) and the predictions of Herwig et al. (2004) computed for AGB stars with $6 M_{\odot}$ HBB (Hot Bottom Burning model) and $2 M_{\odot}$ non-HBB. Like most of the CEMP turnoff 


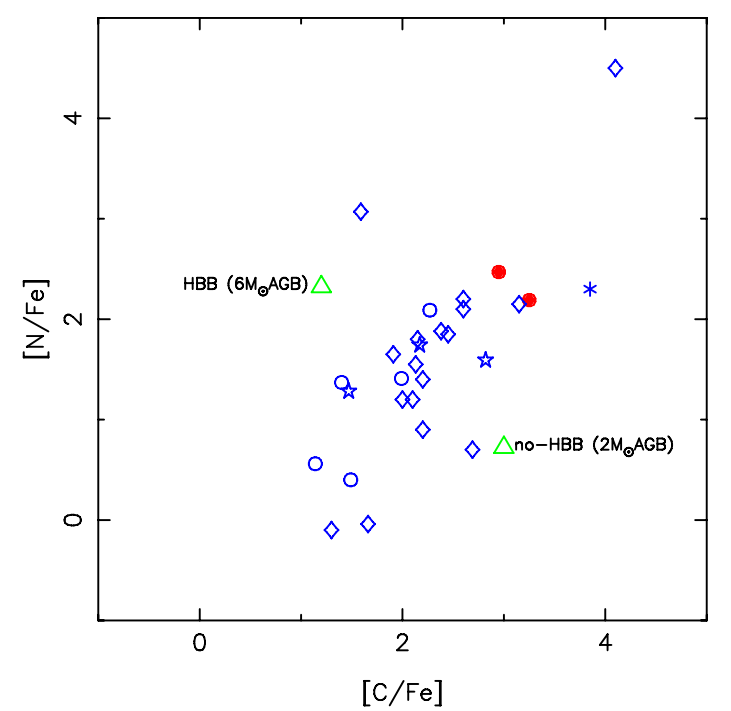

Fig. 5. $[\mathrm{N} / \mathrm{Fe}]$ vs. $[\mathrm{C} / \mathrm{Fe}]$ for CEMP dwarfs and turnoff stars from the recent literature (Sivarani et al. 2006, diamonds), (Behara et al. 2010, stars), (Masseron et al. 2012, open circles), (Christlieb et al. 2002, asterisk), and for our stars SDSS J111407+182831 and SDSS J1143+2020 (filled circles). The AGB models predicted by Herwig et al. (2004), for $[\mathrm{Fe} / \mathrm{H}]=-2.3$ and initial masses $6 M_{\odot}(\mathrm{HBB})$ and $2 M_{\odot}($ non-HBB $)$ are labeled (triangles).

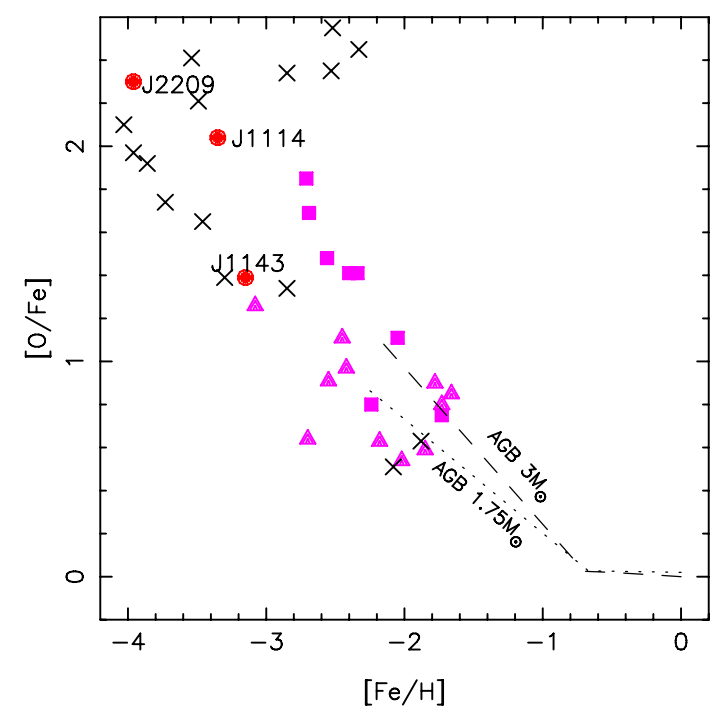

Fig. 6. $[\mathrm{O} / \mathrm{Fe}]$ vs. $[[\mathrm{Fe} / \mathrm{H}]]$ for CEMP-s stars (triangles), CEMP-rs stars (squares), CEMP-no stars (crosses) following Masseron et al. (2010). Our stars are represented by red full circles. The predictions of Karakas \& Lattanzio (2007) for pollution by $1.75 M_{\odot}$ and $3 M_{\odot}$ AGB are indicated as dotted and dashed lines.

stars, SDSS J111407+182831 and SDSS J1143+2020 lie in the region delimited by these two models (see Sivarani et al. 2006).

However, we must have in mind that in a $3 \mathrm{D}$ analysis all stars in this graph would have to be shifted toward lower values of $[\mathrm{C} / \mathrm{Fe}](-0.5 \mathrm{dex})$ and mainly much lower values of $[\mathrm{N} / \mathrm{Fe}]$. This will be discussed in a follow-up paper.

\subsubsection{Heavy elements $\mathrm{Sr}, \mathrm{Ba}, \mathrm{Pb}$, and class determination of the stars}

With a $\mathrm{Ba}$ overabundance $[\mathrm{Ba} / \mathrm{Fe}]>1.0$, the two stars SDSS J111407+182831 and SDSS J1143+2020 belong (Beers $\&$ Christlieb 2005), to the class of the CEMP-s stars or to the

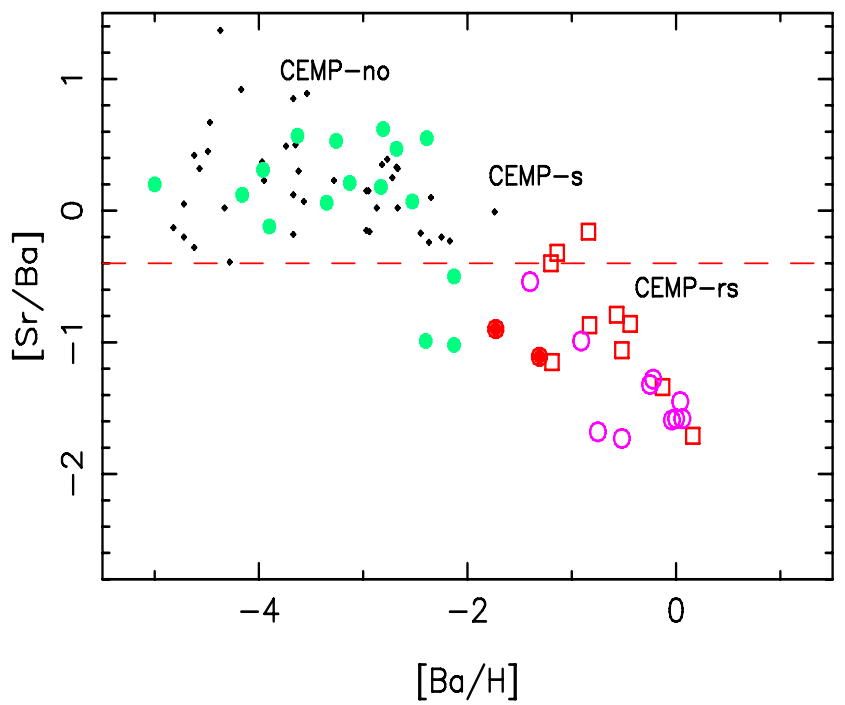

Fig. 7. $[\mathrm{Sr} / \mathrm{Ba}]$ vs. $[\mathrm{Ba} / \mathrm{H}]$ for normal metal-poor stars (black dots) following (Andrievsky et al. 2011), and CEMP stars following Frebel (2010) and Cescutti \& Chiappini (2013). Green filled circles represent CMP-no stars, open red squares CMP-s stars, and open magenta circles CMP-rs stars. The red dots represent SDSS J111407+182831 and SDSS J1143+2020. In case of mass transfer from an AGB companion $[\mathrm{Sr} / \mathrm{Ba}]<-0.4$ (dashed line). Our stars fulfill this requirement.

CEMP-rs stars, depending on the abundance of $\mathrm{Eu}([\mathrm{Ba} / \mathrm{Eu}]>$ 0.5 in CEMP-s stars and $0.0<[\mathrm{Ba} / \mathrm{Eu}]<0.5$ in CEMP-rs stars). The europium line is not visible in these two CEMP turnoff stars, and the low $\mathrm{S} / \mathrm{N}$ of the spectra in the region of the main Europium line $(412.9 \mathrm{~nm})$ does not allow a very restrictive estimate of the Eu abundance.

Masseron et al. (2010) have proposed a classification based on $[\mathrm{Ba} / \mathrm{Fe}]$ alone, when $[\mathrm{Eu} / \mathrm{Fe}]$ is not available. From their Fig. 1, the stars are all CEMP-rs when $[\mathrm{Ba} / \mathrm{Fe}]>2.1$. But when $1.6<[\mathrm{Ba} / \mathrm{Fe}]<1.9$ like in our stars, there is about the same number of CEMP-s and CEMP-rs stars.

On the other hand, Masseron et al. (2010) have shown that, for the same metallicity, the CEMP-rs stars have a higher value of $[\mathrm{O} / \mathrm{Fe}]$ than the CEMP-s stars. In Fig. 6 we have plotted $[\mathrm{O} / \mathrm{Fe}]$ vs. $[\mathrm{Fe} / \mathrm{H}]$ for the stars of Masseron et al. (2010), with $[\mathrm{Fe} / \mathrm{H}]<-1.5$ (their Fig. 18) and for our stars; the dashed and dotted lines in this figure represent the predictions of Karakas \& Lattanzio (2007) for AGB of $1.75 M_{\odot}$ and $3 M_{\odot}$. This graph supports the idea that CEMP-rs stars have been polluted by a higher mass AGB than CEMP-s stars (Masseron et al. 2010) and that SDSS J111407+182831 with its very high oxygen abundance could be classified as a CEMP-rs, but SDSS J1143+2020 as a CEMP-s star.

In Fig. 7 we have plotted $[\mathrm{Sr} / \mathrm{Ba}]$ as a function of $[\mathrm{Ba} / \mathrm{H}]$ for different types of CEMP stars. The abundances of $\mathrm{Sr}$ and Ba have been taken from Frebel (2010). All CMP-s and CMP-rs stars have a known Europium abundance. We adopted the definitions of Sivarani et al. (2006): we called CEMP-no stars all CEMP stars with $[\mathrm{Ba} / \mathrm{Fe}]<1.0$ (CEMP-no or CEMP-low-s), the CEMP-s and CEMP-rs stars have $[\mathrm{Ba} / \mathrm{Fe}]>1.0$ with $[\mathrm{Ba} / \mathrm{Eu}]>0.5$ for the CEMP-s and $0<[\mathrm{Ba} / \mathrm{Eu}]<0.5$ for the CEMP-rs stars. The small dots represent the "normal" EMP stars (Andrievsky et al. 2011). The ratio $[\mathrm{Sr} / \mathrm{Ba}]$ in the CEMP-s and CEMP-rs stars is much lower than in the classical EMP stars. This low ratio is generally compatible with a mass transfer from an AGB companion ( $[\mathrm{Sr} / \mathrm{Ba}]<-0.4)$. In this figure, both SDSS J111407+182831 and SDSS J1143+2020 
M. Spite et al.: Carbon enhanced extremely metal-poor stars
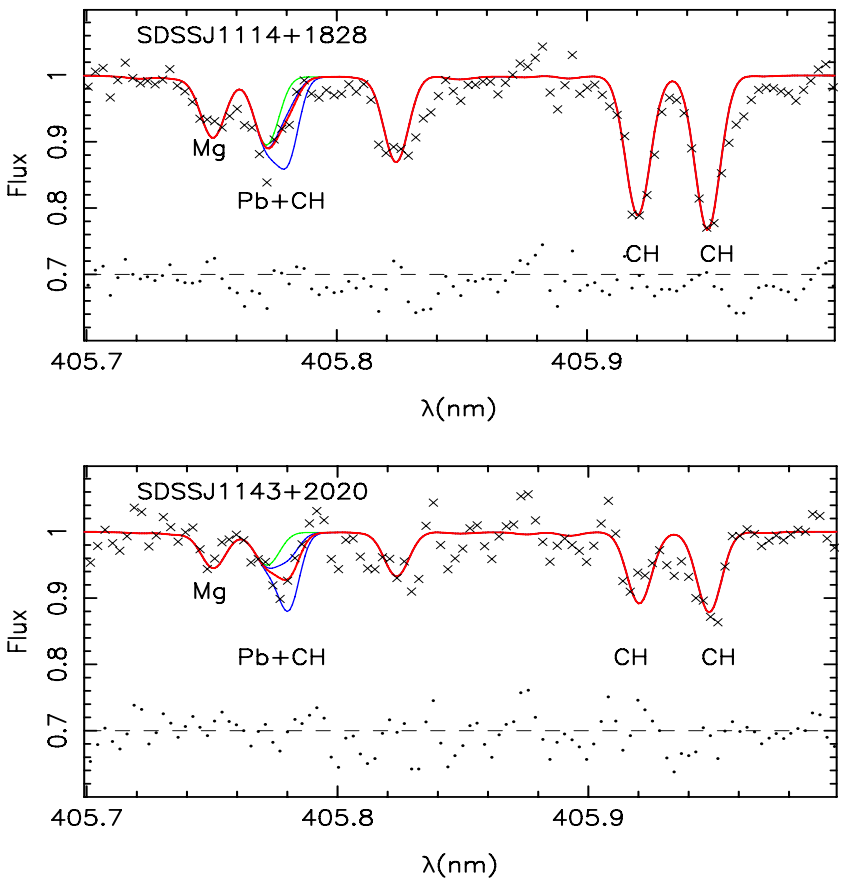

Fig. 8. Spectra of SDSS J1114+1828 and SDSS J1143+2020 in the region of the $\mathrm{Pb}$ line. The synthetic spectra have been computed without $\mathrm{Pb}$ (green line) and with $A(\mathrm{~Pb})=1.5$ and 2.1 (thin blue lines). The best fits (thick red line) are obtained with $A(\mathrm{~Pb})=1.6$ for SDSS J1114+1828 and $A(\mathrm{~Pb})=1.8$ for SDSS J1143+2020. The dots at the bottom of the figures represent the difference between the best fit and the observed spectrum (shifted upward by 0.7 ).

are located in the region corresponding to both the CEMP-s and CEMP-rs stars, their abundances of $\mathrm{Sr}$ and $\mathrm{Ba}$ are compatible with a mass transfer from an AGB (unlike the majority of the CEMP-no stars).

Following Masseron et al. (2010), when $[\mathrm{Fe} / \mathrm{H}]<-3.0$, the CEMP-no stars $([\mathrm{Ba} / \mathrm{Fe}]<+1.0)$ are much more numerous than the CEMP-s or CEMP-rs stars. These authors note that neither CEMP-rs nor CEMP-s stars have been discovered below $[\mathrm{Fe} / \mathrm{H}]<-3.2$. Thus SDSS J111407+182831 with $[\mathrm{Fe} / \mathrm{H}]=$ -3.35 is to date the most metal-poor CEMP-s or CEMP-rs star.

Several carbon-rich stars CEMP-s and CEMP-rs stars are also lead-rich (see e.g.: Cohen et al. 2003; Barbuy et al. 2005; Bisterzo et al. 2006). We tried to detect Pb in SDSS J1114 +1828 and SDSS J1143+2020 (Fig. 8). The best fits were obtained with $A(\mathrm{~Pb})=1.6 \pm 0.3$ for SDSS J1114+1828, and $A(\mathrm{~Pb})=1.8 \pm 0.2$ for SDSS J1143+2020. The corresponding equivalent widths are respectively about 4 and $6 \mathrm{~mA}$. In this region of the spectrum the $\mathrm{S} / \mathrm{N}$ is around 50 (see Table 2), therefore from the Cayrel (1988) formula, the error on the equivalent width is lower than $2 \mathrm{~m} \AA$. For SDSS J1143+2020 the detection of $\mathrm{Pb}$ is highly significant $(>95 \%)$. In both stars $[\mathrm{Pb} / \mathrm{Fe}] \approx+3.0$, a value very close to the values of $[\mathrm{Pb} / \mathrm{Fe}]$ generally found from LTE computations in similar stars (Bisterzo et al. 2006). But the $\mathrm{Pb}$ abundance has to be corrected for NLTE effects which, following Mashonkina et al. (2012), are strong in metal-poor stars. These authors have not computed the correction for turnoff stars with a metallicity as low as those of SDSS J1114+1828 and SDSS J1143+2020, but since the correction increases with the temperature and with decreasing metallicity, it is at least equal to +0.3 according to their Table 1.

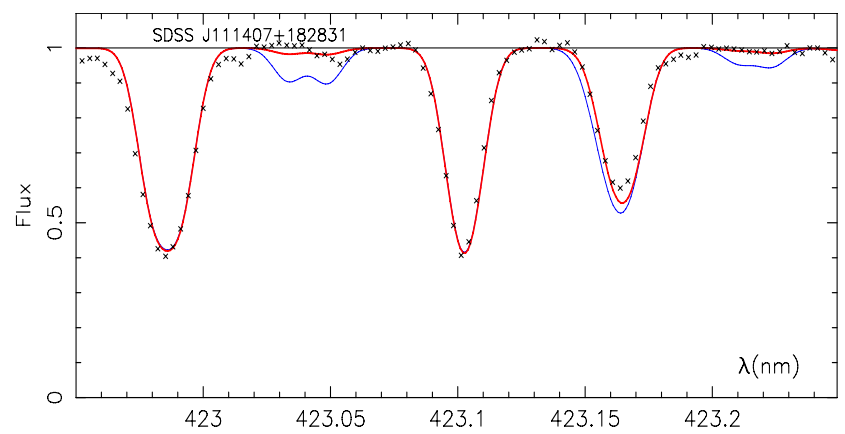

Fig. 9. UVES spectrum in the region of the $\mathrm{CH}$ band in SDSS J111407+182831 compared to theoretical spectra computed with $A\left({ }^{12} \mathrm{C}\right)=8.4$ and ${ }^{12} \mathrm{C} /{ }^{13} \mathrm{C}$ equal to either 10 (thin blue line) or 60 (thick red line). The abscissa is the wavelength in $\mathrm{nm}$, and the ordinate the relative flux. The ${ }^{13} \mathrm{C}$ feature is not visible in the observed spectrum, and we derived for this $\operatorname{star}{ }^{12} \mathrm{C} /{ }^{13} \mathrm{C} \geq 60$.

\subsection{2. ${ }^{12} \mathrm{C} /{ }^{13} \mathrm{C}$ ratio}

The ${ }^{13} \mathrm{C}$ features are not visible in the observed spectra (Fig. 9). We deduced lower limits: ${ }^{12} \mathrm{C} /{ }^{13} \mathrm{C} \geq 60$ for SDSS J111407+182831 and ${ }^{12} \mathrm{C} /{ }^{13} \mathrm{C} \geq 22$ for SDSS J1143+2020. An example of the ${ }^{13} \mathrm{C}$ feature is displayed in Fig. 9. These lower limits are compatible with the values found in the CEMP-s and CEMP-rs stars (see Masseron et al. 2010, their Fig. 17). Masseron et al. (2010) have remarked that, although the mechanism responsible for the $\mathrm{N}$ production may be attributed to $\mathrm{CBB}$ (cool bottom burning) in the framework of AGB evolution, no current AGB models reproduce the observed trend of $\log ^{12} \mathrm{C} /{ }^{13} \mathrm{C}$ vs. [C/N].

\subsubsection{Lithium abundance}

In normal (non $\mathrm{C}$-enhanced) turnoff stars with a metallicity higher than $[\mathrm{Fe} / \mathrm{H}]=-3.0$, the lithium abundance $A(\mathrm{Li})$ is generally constant, independent of the temperature and metallicity and equal to about 2.2 dex defining a plateau (see e.g. Spite et al. $2012 \mathrm{~b}$ ). But below $[\mathrm{Fe} / \mathrm{H}]=-3.0$ there is a meltdown of the plateau (e.g. Bonifacio et al. 2007; González Hernández et al. 2008; Sbordone et al. 2010) and a large part of the normal very deficient stars have a lithium abundance lower than 2.2.

In the CEMP turnoff stars, even with a metallicity higher than $[\mathrm{Fe} / \mathrm{H}]=-3.0$, the lithium abundance is often lower than the value of the plateau. In the spectra of SDSS J1114+1828 and SDSS J1143+2020 the lithium line is not visible (Fig. 10). We derived in both cases $A(\mathrm{Li}) \leq 1.5$, a lithium abundance well below the value of the plateau.

The low abundance of Li in CEMP ( $\mathrm{s}$ and rs) stars is discussed by Masseron et al. (2012). Briefly, before the transfer of material from the AGB companion, the lithium abundance in the observed star should be the same as in normal metal-poor turnoff stars, but as more and more AGB material is transferred to the atmosphere of the star, the carbon abundance increases while the lithium abundance decreases because in most cases the lithium abundance in the AGB star is lower than the lithium plateau.

\subsection{SDSS J220924-002859}

SDSS J220924-002859 with $[\mathrm{Fe} / \mathrm{H}] \approx-4$ is the most metal-poor star of our sample. Compared to the other two stars its carbon abundance is also ten times lower. But it is also the faintest star of our sample (Table 1) and the $\mathrm{S} / \mathrm{N}$ of the spectra is much lower 

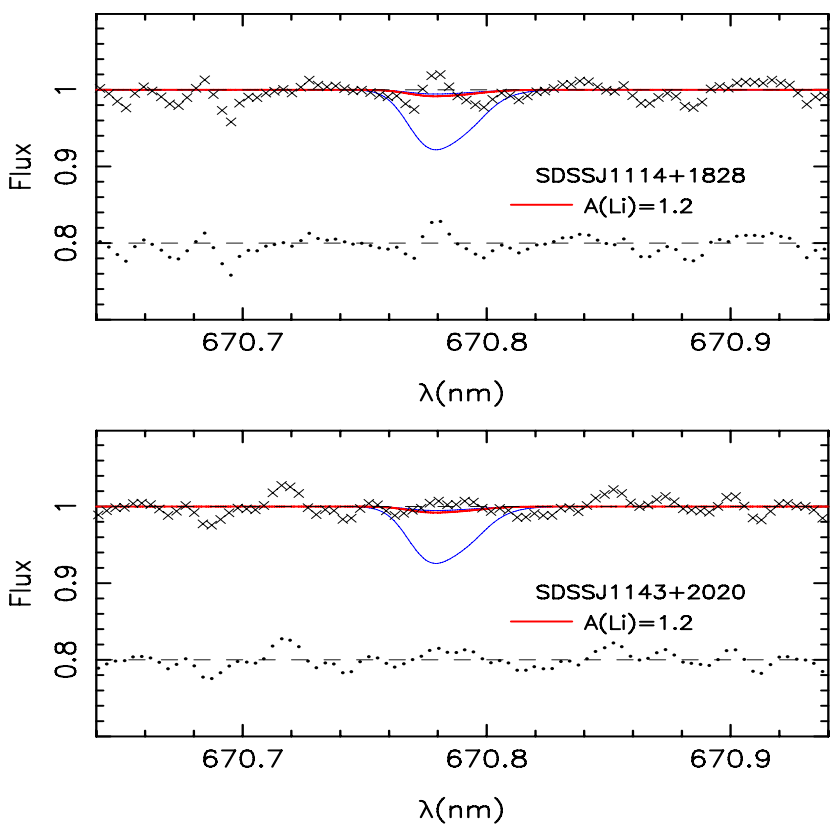

Fig. 10. UVES spectrum in the region of the lithium feature. The thin blue line corresponds to the lithium feature computed with the classical abundance $A(\mathrm{Li})=2.2$, and the thick red line to the best fit $(A(\mathrm{Li})=1.2$ in both cases). The dots at the bottom of the figures represent the difference between this best fit and the observed spectrum (shifted upward by 0.8$)$. We adopted in both cases $A(\mathrm{Li})<1.5$.

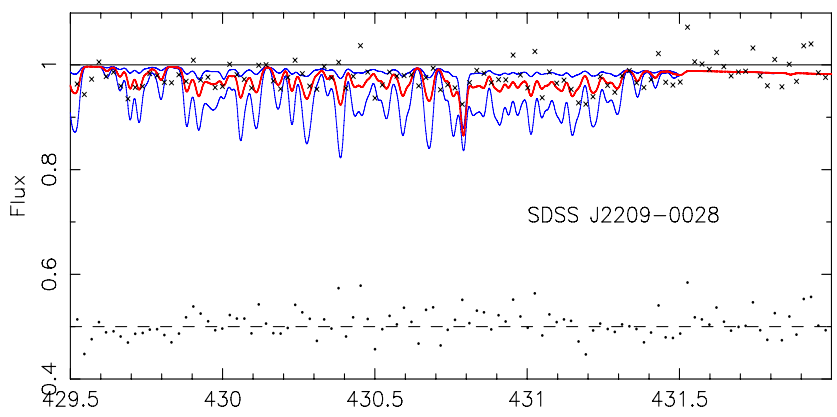

Fig. 11. UVES spectrum in the region of the CH band in SDSS J22090028. The thin blue lines correspond to the computation of the $\mathrm{CH}$ band with an abundance $A(\mathrm{C})=6.5$ and 7.5. The thick red line corresponds to $A(C)=7.1$ (best fit) and the dots at the bottom of the figure represent the difference between the synthetic spectrum computed with $A(C)=7.1$ and the observed spectrum (shifted upward by 0.5 ).

(Table 2). As a consequence the abundance of only few elements could be measured in this star.

The 1D carbon abundance in SDSS J220924-002859 was estimated to be $A(C)=7.0 \pm 0.2$ dex (Fig. 11), with the 3D correction we derived $A(\mathrm{C})=6.5 \pm 0.2$ dex. However, we remark that the $\mathrm{S} / \mathrm{N}$ in this star is so poor that the $\mathrm{CH}$ band in the spectrum is at the limit of the detection and it would be very interesting to obtain a new better spectrum in this region.

With $[\mathrm{O} / \mathrm{Fe}]=2.10$, SDSS J220924-002859 is the most oxygen-rich star in our sample (Fig. 12). The barium line is not visible in our spectrum and we derived, within the LTE hypothesis, an upper limit $[\mathrm{Ba} / \mathrm{Fe}]<0.75$ (this upper limit becomes $[\mathrm{Ba} / \mathrm{Fe}]<1.05$ when the NLTE effects are taken into account). It should thus belong to the CEMP-no stars (defined by $[\mathrm{Ba} / \mathrm{Fe}]_{\text {LTE }}<+1$ see also the Fig. 7 ).

The star is very oxygen-rich, and in Fig. 6 it indeed appears in the region of the CEMP-no stars. SDSS J220924-002859

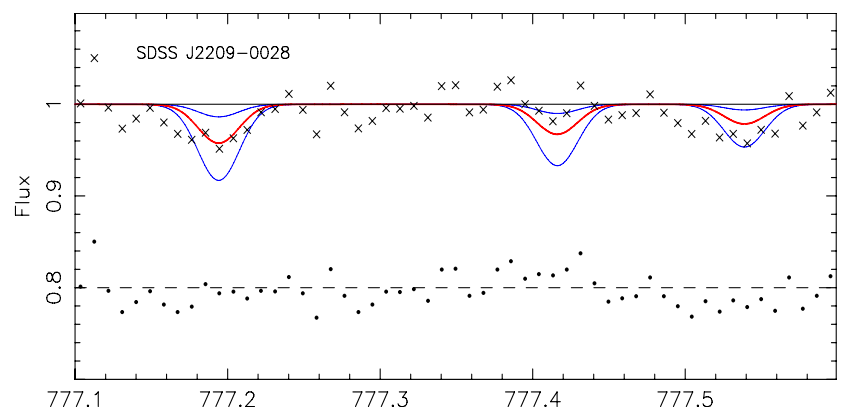

Fig. 12. Thin blue lines correspond to synthetic spectra computed with $A(\mathrm{O})=6.5$ and 7.5. and the thick red line (best fit) has been obtained with $A(\mathrm{O})=7.1$. The crosses represent the observed spectrum (binned x2). The dots at the bottom of the figure are the difference between the best fit and the observed spectrum (shifted upward by 0.8 ).

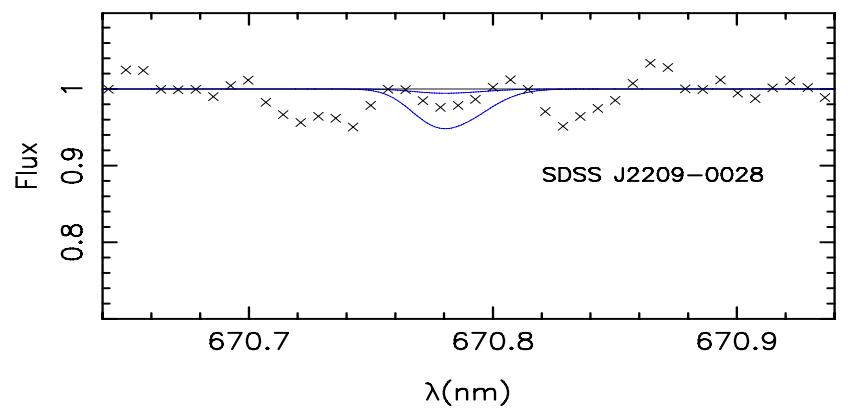

Fig. 13. UVES spectrum in the region of the lithium feature. The thin blue lines correspond to the lithium feature computed with the abundances $A(\mathrm{Li})=1.2$ and 2.2. The noise is important in this region of the spectrum and therefore we derive $A(\mathrm{Li}) \leq 2.2$.

belongs to this class, as do most of the extremely iron-poor carbon-rich stars (Masseron et al. 2010).

In SDSS J220924-002859 the best fit is obtained with $A(\mathrm{Li})=1.8 \pm 0.4$ (Fig. 13). The noise is very high in this region of the spectrum and the abundance of the plateau $A(\mathrm{Li})=2.2$ cannot be excluded.

\section{Discussion and conclusion}

In Fig. 14 we plotted the recent measurements of the abundance of $\mathrm{C}$ in CEMP dwarf and turnoff stars as a function of $[\mathrm{Fe} / \mathrm{H}]$, for $[\mathrm{Fe} / \mathrm{H}]<-1.8$ collecting the data of Sivarani et al. (2006, their Table 4), Frebel et al. (2005, 2007), Thompson et al. (2008), Aoki et al. (2009), Behara et al. (2010), Placco et al. (2011), Carollo et al. (2011), Masseron et al. $(2010,2012)$ Yong et al. (2013b), and this paper. We did not considered the giants because their carbon abundance can be affected by mixing with deep layers (see e.g., at low metallicity, Spite et al. 2005, 2006). In this figure we have kept the carbon abundance deduced from the $\mathrm{CH}$ molecular band and 1D computations to facilitate the comparison with the results in the literature since generally these previous abundances were derived from this feature and from 1D models. All stars in the graph are turnoff stars or dwarfs, the 3D correction must be about the same for all these stars, and, after this correction, the global view should remain similar.

According to the definition of the carbon-rich metal-poor stars $([\mathrm{C} / \mathrm{Fe}]>+1)$ these stars are located above the dashed blue line in Fig. 14. From this figure it seems that for $[\mathrm{Fe} / \mathrm{H}]>-3$ the logarithm of the carbon abundance $A(\mathrm{C})$ is almost constant close to 8.25 , a value slightly lower than the solar value $(A(C)=8.5)$. This can reflect the fact that the carbon quantity transferred by 


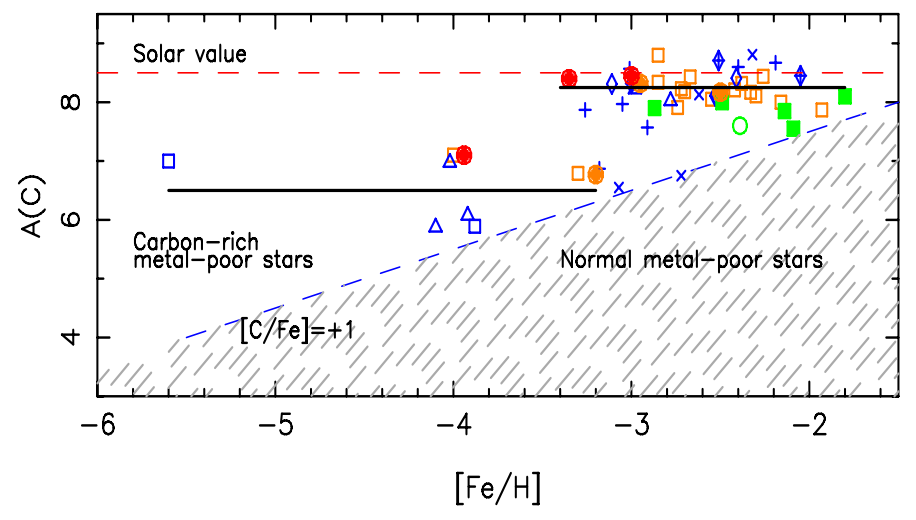

Fig. 14. Abundance of carbon $A(\mathrm{C})$ vs. $[\mathrm{Fe} / \mathrm{H}]$ in dwarfs and turnoff CEMP stars, following Sivarani et al. (2006, their Table 4), [orange open squares]), Frebel et al. (2005, 2007) [blue open squares], Thompson et al. (2008) [green open circle], Aoki et al. (2009) [blue open diamonds], Behara et al. (2010) [full orange circles], Placco et al. (2011) [blue $\times$ ), Carollo et al. (2011) [blue +], Masseron et al. (2010, 2012) [full green squares], Yong et al. (2013b) [blue open triangles]. Our measurements are represented with full red circles. The dashed blue line (representing $[\mathrm{C} / \mathrm{Fe}]=+1$ ) separates the region of the carbonrich metal-poor stars from the region of the normal metal-poor stars (hatched). When $[\mathrm{Fe} / \mathrm{H}]$ is higher than -3 the carbon abundance is almost constant and close to 8.25 (see also Masseron et al. 2010). For $[\mathrm{Fe} / \mathrm{H}]<-3.4$ the carbon abundance drops suddenly to about 6.5 . However, it is difficult to decide whether at very low metallicity $A(\mathrm{C})$ is also constant, or whether the line $A(C)=6.5$ represents an upper limit of the carbon abundance in these extremely metal-poor star (Masseron et al. 2010).

the defunct AGB companion to the observed carbon-rich metalpoor star, has been such as to reach the same total amount in all stars, whatever the metallicity between -1.8 and -3 (see Masseron et al. 2010).

The carbon abundance is clearly lower, with $A(\mathrm{C}) \approx 6.8$ for the low metallicities $([\mathrm{Fe} / \mathrm{H}]<-3.4)$. None of these stars have a (1D) carbon abundance significantly higher than $A(C)=$ 7.0. However, the number of carbon-rich metal-poor stars with $[\mathrm{Fe} / \mathrm{H}]<-3.4$ is very small and it is not possible to decide wether the mean value $A(C)=6.8$ represents a plateau of the carbon abundance or if it is an upper limit. Moreover, in this metallicity range, the stars are generally faint and a precise determination of the carbon abundance below $A(\mathrm{C})=7.0$ is difficult in turnoff stars. It would be interesting to find more CEMP dwarf stars with a temperature below $6000 \mathrm{~K}$ and consequently a stronger $\mathrm{CH}$ absorption band, to reach a better determination of the behavior of the carbon abundance in the extremely metal-poor carbon stars.

In all stars with $[\mathrm{Fe} / \mathrm{H}]<-3.4$, the barium abundance is also rather low: $[\mathrm{Ba} / \mathrm{Fe}]<1.0$. All these stars belong to the class of the CEMP-no stars. Five other stars are located close to this plateau but with a metallicity higher than $[\mathrm{Fe} / \mathrm{H}]=-3$.4. In two of them (studied from high-resolution spectra) the abundance of Ba was measured. In BPS CS 29528-041, [Ba/Fe] is lower than 1.0. Therefore, this star belongs to the CEMP-no stars (Sivarani et al. 2006), but SDSS J1036+1212 (Behara et al. 2010) is Ba-rich $([\mathrm{Ba} / \mathrm{Fe}]=1.17)$ and has a ratio $[\mathrm{Eu} / \mathrm{Fe}]=1.26$. In this star $[\mathrm{Ba} / \mathrm{Eu}]=-0.09$, this value is very close to $[\mathrm{Ba} / \mathrm{Eu}]=0.0$ (within the error bar) and thus SDSS J1036+1212 is probably a CEMP-rs star (see also the Fig. 1 of Masseron et al. 2010).

The cause of the abundance anomalies of the CEMP-no stars are currently only poorly understood. An enrichment in only C, $\mathrm{N}$, and $\mathrm{O}$ by AGBs has been proposed (Masseron et al. 2010), but in this case we would expect that many CEMP-no stars are binaries: only one CEMP-no star is known to be a binary (Preston \& Sneden 2001), and the radial velocity of SDSS J220924002859 seems to be stable between 2003 and 2011 (see Sect. 3). However, more radial velocity measurements over a longer period of time would be necessary to exclude binarity. The abundance pattern of the CEMP-no stars could be also the result of a pre-enrichment of the interstellar medium by e.g. faint supernovae associated with the first generations of stars (Umeda et al. 2003, 2005; Tominaga et al. 2007), or by C-rich winds of massive rotating EMP stars (Hirschi et al. 2006; Meynet et al. 2006).

To better interpret the abundance pattern of the CEMP-no stars, it is important to clearly establish wether the carbon abundance in this type of star is constant at a level of $A(C) \approx 6.8$ or wether this value is only an upper limit of the carbon abundance. It would be very important to significantly enlarge the sample of the abundance pattern of the very metal-poor CEMP stars and to study the scatter around the upper plateau, as well as around the possible lower plateau. Monitoring the radial velocities of the CEMP-no stars would also be important to research the origin of the CEMP stars.

A 3D analysis of the $\mathrm{CH}$ and $\mathrm{CN}$ bands suggests that in metal-poor turnoff stars the carbon abundance could be 0.7 dex lower and the nitrogen abundance 2.4 dex lower than those derived in a 1D analysis of the $\mathrm{CH} \mathrm{G}$ band or the $\mathrm{CN}$ band. This effect, which strongly depends on the external layers of the models, will be studied in detail in a forthcoming paper.

Acknowledgements. The authors thanks the referee for his/her very constructive remarks that helped us improve our manuscript.They thank David Yong for providing data in advance of publication This work has been supported by the "Programme National de Physique Stellaire" and the "Programme National de Cosmologie et Galaxies" (CNRS-INSU). E.C., N.C., and H.G.L. acknowledge financial support by the Sonderforschungsbereich SFB 881 "The Milky Way System (subprojects A4 and A5)" of the German Research Foundation (DFG).

\section{References}

Abazajian, K. N., Adelman-McCarthy, J. K., Agüeros, M. A., et al. 2009, ApJS, 182,543

Alvarez, R., \& Plez, B. 1998, A\&A, 330, 1109

Asplund, M. 2005, ARAA, 43, 481

Andrievsky, S. M., Spite, M., Korotin, S. A., et al. 2007, A\&A, 464, 1081 Andrievsky, S. M., Spite, M., Korotin, S. A., et al. 2008, A\&A, 481, 481 Andrievsky, S. M., Spite, M., Korotin, S. A., et al. 2009, A\&A, 494, 1083 Andrievsky, S. M., Spite, F., Korotin, S. A., et al. 2011, A\&A, 530, A105

Aoki, W., Frebel, A., Christlieb, N., et al. 2006, ApJ, 639, 897

Aoki, W., Beers, T. C., Sivarani, T., et al. 2008, ApJ, 678, 1351

Barbuy, B., Spite, M., Spite, F., et al. 2005, A\&A, 429, 1031

Barklem, P. S., Belyaev, A. K., Guitou, M., \& Feautrier, N. 2012, A\&A, 541, A80

Beers, T. C., \& Christlieb, N. 2005, ARA\&A, 43, 531

Behara, N. T., Bonifacio, P., Ludwig, H., et al. 2010, A\&A, 513, A72

Bergemann, M., \& Cescutti, G. 2010, A\&A, 522, A9

Bergemann, M., \& Gehren, T. 2008, A\&A, 492, 823

Bisterzo, S., Gallino, R., Straniero, O., et al. 2006, Mem. S.A.It. 77, 985

Bonifacio, P., Molaro, P., Sivarani, T., et al. 2007, A\&A, 462, 851

Bonifacio, P., Spite, M., Cayrel, R., et al. 2009, A\&A, 501, 519

Caffau, E., \& Ludwig, H.-G. 2007, A\&A, 467, L11

Caffau, E., Ludwig, H.-G., Steffen, M., et al. 2008, A\&A, 488, 1031

Caffau, E., Bonifacio, P., François, P., et al. 2011a, Nature, 477, 67

Caffau, E., Bonifacio, P., François, P., et al. 2011b, A\&A, 534, A4

Caffau, E., Ludwig, H.-G., Steffen, M., Freytag, B., \& Bonifacio, P. 2011c, Sol. Phys., 268, 255

Caffau, E., Bonifacio, P., François, P., et al. 2012, A\&A, 542, A51

Carollo, D., Beers, T. C., Bovy, J., et al. 2012, ApJ, 744, 195

Cayrel, R. 1988, The impact of Very High S/N Spectroscopy on Stellar Physics, eds. G. Cayrel de Strobel, \& M. Spite (Kluwer Academic Publishers), Proc. IAU Symp., 132, 345

Cayrel, R., Depagne, E., Spite, M., et al. 2004, A\&A, 416, 1117 
Cescutti, G., \& Chiappini, C. 2013, Proc. XII International symposium on Nuclei in the Cosmos, eds. J. Lattanzio, A. Karakas, M. Lugaro, \& G. Dracoulis, http://pos.sissa.it [arXiv: 1301.1908]

Christlieb, N., Bessell, M. S., Beers, T. C., et al. 2002, Nature, 419, 904

Cohen, J. G., Christlieb, N., Qian, Y.-Z., et al. 2003, ApJ, 588, 1082

Collet, R., Asplund, M., \& Trampedach, R. 2007, A\&A, 469, 687

Dekker, H., D’Odorico, S., Kaufer, A., Delabre, B., \& Kotzlowski, H. 2000, Proc. SPIE, 4008, 534

Frebel, A. 2010, AN, 331, 474

Frebel, A., Aoki, W., Christlieb, N., et al. 2005, Nature, 434, 871

Frebel, A., Christlieb, N., Norris, J. E., et al. 2006, ApJ, 652, 1585

Frebel, A., Johnson, J. L., \& Bromm, V. 2007, MNRAS, 380, L40

Freytag, B., Steffen, M., Ludwig, H.-G., et al. 2012, J. Comp. Phys., 231, 919

Gehren, T., Liang, Y. C., Shi, J. R., et al. 2004, A\&A, 413, 1045

González Hernández, J. I., Bonifacio, P., Ludwig, H.-G., et al. 2008, A\&A, 480, 233

González Hernández, J. I., Bonifacio, P., Ludwig, H.-G., et al. 2010, A\&A, 519, A46

Gustafsson, B., Bell, R. A., Eriksson, K., \& Nordlund, Å. 1975, A\&A, 42, 407

Gustafsson, B., Edvardsson, B., Eriksson, K., et al. 2003, in Stellar Atmosphere Modeling, eds. I. Hubeny, D. Mihalas, \& K. Werner, ASP Conf. Ser., 288, 331

Gustafsson, B., Edvardsson, B., Eriksson, K., et al. 2008, A\&A, 486, 951

Herwig, F. 2004, ApJS, 155, 651

Hill, V., Plez, B., Cayrel, R., et al. 2002, A\&A, 387, 560

Hirschi, R., Fröhlich, C., Liebendörfer, M., \& Thielemann F.-K. 2005, RvMA, 19, Proc. 79th Annual Scientific Meeting of the Deutsche Astronomische Gesellschaft, 101 [arXiv: astro-ph/0601502]

Karakas, A., \& Lattanzio, J. C. 2007, PASA, 24, 103

Lai, D. K., Bolte, M., Johnson, J. A., et al. 2008, ApJ, 681, 1524

Lodders, K., Palme, H., \& Gail, H.-P. 2009, in Landolt-Börnstein, New Series, Volume VI/4B Chapt. 4.4, ed. J. E. Trümper (Berlin, Heifelberg, New York: Springer-Verlag), 560

Lucatello, S., Tsangarides, S., Beers, T. C., et al. 2005, ApJ, 625, 825

Lucatello, S., Beers, T. C., Christlieb, N., et al. 2006, ApJ, 652, L37

Ludwig, H.-G. 1992, University of Kiel, Ph.D. Thesis

Ludwig, H.-G., Bonifacio, P., Caffau, E., et al. 2008, Phys. Scr., 133, 014037

Ludwig, H.-G., Caffau, E., Steffen, M., et al. 2009, Mem. Soc. Astron. It., 80, 711
Marsteller, B., Beers, T. C., Rossi, S., et al. 2005, Nucl. Phys. A, 758, 312

Mashonkina, L. 2013, A\&A, 550, A28

Mashonkina, L., Gehren, T., \& Bikmaev, I. 1999, A\&A, 343, 519

Mashonkina, L., Ryabtsev, A., \& Frebel, A. 2012, A\&A, 540, A98

Masseron, T., Johnson, J. A., Plez, B., et al. 2010, A\&A, 509, A93

Masseron, T., Johnson, J. A., Lucatello, S., et al. 2012, ApJ, 751, 14

Meynet, G., Ekström, S., \& Maeder, A. 2006, A\&A, 447, 623

Nordlund, A. 1983, in Solar and stellar magnetic fields: Origins and coronal effects; Proc. Symposium, Zurich, Switzerland, August 2-6, 1982 (A84-42426 20-90) (Dordrecht: D. Reidel Publishing Co.), 79

Paunzen, E., Kamp, I., Iliev, I. Kh., et al. 1999, A\&A, 345, 597

Placco, V. M., Kennedy, C. R., Beers, T. C., et al. 2011, ApJ, 142, 188

Plez, B., \& Cohen, J. G. 2005, A\&A, 434, 1117

Plez, B., Masseron, T., Van Eck, S., et al. 2008, in 14th Cambridge Workshop on Cool Star, Stellar Systems, and the Sun, ed. G. van Belle, ASP Conf. Ser., 384, CD-rom

Plez, B. 2012, ascl.soft05004P

http: //adsabs . harvard.edu/abs/2012ascl. soft05004P

Preston, G. W., \& Sneden, C. 2001, AJ, 122, 1545

Rossi, S., Beers, T. C., \& Sneden, C. 1999, in The Third Stromlo Symposium: The Galactic Halo, eds. B. K. Gibson, R. S. Axelrod, \& M. E. Putman (San Francisco: ASP), ASP Conf. Ser., 165, 264

Sbordone, L., Bonifacio, P., Caffau, E., et al. 2010, A\&A, 522, A26

Schlegel, D. J., Finkbeiner, D. P., \& Davis, M. 1998, ApJ, 500, 525

Sivarani, T., Beers, T. C., Bonifacio, P., et al. 2006, A\&A, 459, 125

Spite, M., Cayrel, R., Plez, B., et al. 2005, A\&A, 430, 655

Spite, M., Cayrel, R., Hill, V., et al. 2006, A\&A, 455, 291

Spite, M., Andrievsky, S. M., Spite, F., et al. 2012a, A\&A, 541, A143

Spite, M., Spite, F., \& Bonifacio, P. 2012b, Mem. Soc. Astron. It. Suppl., 22, in press

Steenbock, W., \& Holweger, H. 1984, A\&A, 130, 319

Stürenburg, S., \& Holweger, H. 1990, A\&A, 237, 125

Thompson, I. B., Ivans, I. I., Bisterzo, S., et al. 2008, ApJ, 677, 556

Tominaga, N., Umeda, H., \& Nomoto, K. 2007, ApJ, 660, 516

Umeda, H., \& Nomoto, K. 2003, Nature, 422, 871

Umeda, H., \& Nomoto, K. 2005, ApJ, 619, 427

Yong, D., Norris, J. E., Bessell, M. S., et al. 2013a, ApJ, 762, 27

Yong, D., Norris, J. E., Bessell, M. S., et al. 2013b, ApJ, 762, 26

York, D. G., Adelman, J., Anderson, J. E., Jr., et al. 2000, AJ, 120, 1579 
M. Spite et al.: Carbon enhanced extremely metal-poor stars

Table 5. Linelist equivalent widths and abundances.

\begin{tabular}{|c|c|c|c|c|c|c|c|c|c|c|c|c|}
\hline \multirow{2}{*}{ Elem } & \multirow[b]{2}{*}{$\begin{array}{c}\lambda \\
(\AA)\end{array}$} & \multirow[b]{2}{*}{$\begin{array}{r}\text { Exc. Pot. } \\
(\mathrm{eV})\end{array}$} & \multirow[b]{2}{*}{$\log g f$} & \multicolumn{3}{|c|}{ SDSS J111407+182831 } & \multicolumn{3}{|c|}{ SDSS J114323+202058 } & \multicolumn{3}{|c|}{ SDSS J220924-002859 } \\
\hline & & & & $\begin{array}{r}E W \\
(\mathrm{~m} \AA)\end{array}$ & $\begin{array}{r}\text { abund } \\
\text { LTE }\end{array}$ & $\begin{array}{l}\text { abund } \\
\text { NLTE }\end{array}$ & $\begin{array}{r}E W \\
(\mathrm{~m} \AA)\end{array}$ & $\begin{array}{r}\text { abund } \\
\text { LTE }\end{array}$ & $\begin{array}{l}\text { abund } \\
\text { NLTE }\end{array}$ & $\begin{array}{c}E W \\
(\mathrm{~m} \AA)\end{array}$ & $\begin{array}{r}\text { abund } \\
\text { LTE }\end{array}$ & $\begin{array}{l}\text { abund } \\
\text { NLTE }\end{array}$ \\
\hline $\mathrm{C} 1$ & 4932.049 & 7.69 & -1.884 & syn & 8.40 & 7.97 & syn & 8.20 & 7.77 & & & \\
\hline O 1 & 7771.941 & 9.15 & 0.369 & syn & & & syn & 7.20 & 7.07 & syn & 7.10 & 6.97 \\
\hline O 1 & 7774.161 & 9.15 & 0.223 & syn & 7.45 & 7.31 & syn & 6.90 & 6.82 & syn & 7.10 & 6.97 \\
\hline O 1 & 7775.388 & 9.15 & 0.001 & syn & & & & & & & & \\
\hline $\mathrm{Na} 1$ & 5889.951 & 0.00 & 0.117 & 149.8 & 4.67 & 4.57 & 142.6 & 4.63 & 4.53 & & & \\
\hline Na 1 & 5895.924 & 0.00 & -0.184 & 127.2 & 4.69 & 4.59 & 122.0 & 4.66 & 4.56 & & & \\
\hline $\operatorname{Mg} 1$ & 3829.355 & 2.71 & -0.231 & syn & 5.35 & 5.49 & syn & 4.90 & 5.00 & syn & 3.65 & 3.90 \\
\hline $\mathrm{Mg} 1$ & 3832.304 & 2.71 & 0.146 & syn & 5.50 & 5.64 & syn & 5.05 & 5.15 & syn & 3.95 & 4.20 \\
\hline $\mathrm{Mg} 1$ & 3838.290 & 2.72 & 0.415 & syn & 5.55 & 5.69 & syn & 5.05 & 5.15 & syn & 3.95 & 4.20 \\
\hline $\mathrm{Mg} 1$ & 4702.991 & 4.35 & -0.666 & 39.3 & 5.64 & 5.78 & 14.5 & 5.11 & 5.21 & & & \\
\hline Al 1 & 3961.520 & 0.01 & -0.323 & 48.0 & 2.83 & 3.48 & 31.5 & 2.55 & 3.20 & & & \\
\hline $\mathrm{Ca} 1$ & 4226.728 & 0.00 & 0.244 & 81.2 & 3.30 & 3.42 & 87.8 & 3.49 & 3.64 & 42.1 & 2.60 & 2.82 \\
\hline $\mathrm{Ca} 1$ & 4454.779 & 1.90 & 0.258 & 17.2 & 3.57 & 3.70 & 16.7 & 3.54 & 3.70 & & & \\
\hline $\mathrm{Ca} 1$ & 6162.173 & 1.90 & -0.090 & 5.4 & 3.25 & 3.43 & 13.6 & 3.71 & 3.89 & & & \\
\hline $\mathrm{Ca} 2$ & 3933.680 & 0.00 & 0.105 & syn & 3.45 & 3.44 & syn & 3.55 & 3.55 & syn & 2.85 & 2.82 \\
\hline $\mathrm{Ca} 2$ & 8498.023 & 1.69 & -1.416 & 118.2 & 3.65 & 3.30 & 133.0 & 3.87 & 3.55 & 92.6 & 3.35 & 3.05 \\
\hline $\mathrm{Ca} 2$ & 8542.091 & 1.70 & -0.463 & 206.4 & 3.50 & 2.99 & 250.0 & 3.74 & 3.19 & 180.6 & 3.47 & 2.87 \\
\hline $\mathrm{Ca} 2$ & 8662.170 & 1.69 & -0.723 & 211.3 & 3.76 & 3.24 & 215.0 & 3.82 & 3.29 & 134.5 & 3.36 & 2.81 \\
\hline Sc 2 & 4246.822 & 0.31 & 0.242 & 47.2 & 0.71 & & 25.7 & 0.27 & & & & \\
\hline Ti 1 & 4981.731 & 0.85 & 0.504 & syn & 2.1 & & & & & & & \\
\hline Ti 2 & 3759.291 & 0.61 & 0.280 & syn & 1.7 & & 70.4 & 2.03 & & & & \\
\hline Ti 2 & 3761.320 & 0.57 & 0.180 & syn & 1.7 & & 67.4 & 2.02 & & & & \\
\hline $\mathrm{Ti} 2$ & 3913.461 & 1.12 & -0.420 & 16.0 & 1.81 & & 20.0 & 1.95 & & & & \\
\hline Ti 2 & 4417.714 & 1.16 & -1.190 & syn & 2.1 & & 5.2 & 2.05 & & & & \\
\hline $\mathrm{Ti} 2$ & 4443.794 & 1.08 & -0.720 & 12.4 & 1.90 & & 17.1 & 2.08 & & & & \\
\hline $\mathrm{Ti} 2$ & 4450.482 & 1.08 & -1.520 & syn & 2.0 & & 5.1 & 2.29 & & & & \\
\hline Ti 2 & 4464.449 & 1.16 & -1.810 & & & & 4.3 & 2.57 & & & & \\
\hline Ti 2 & 4468.507 & 1.13 & -0.600 & 19.1 & 2.05 & & 14.7 & 1.93 & & & & \\
\hline $\mathrm{Ti} 2$ & 4501.270 & 1.12 & -0.770 & 10.3 & 1.88 & & 10.1 & 1.89 & & & & \\
\hline $\mathrm{Ti} 2$ & 4533.960 & 1.24 & -0.530 & syn & 1.75 & & 19.1 & 2.09 & & & & \\
\hline Ti 2 & 4563.757 & 1.22 & -0.690 & 8.4 & 1.80 & & 8.1 & 1.80 & & & & \\
\hline Ti 2 & 4571.968 & 1.57 & -0.320 & syn & 1.8 & & 20.4 & 2.23 & & & & \\
\hline V 1 & 4379.230 & 0.30 & 0.580 & 10.8 & 2.25 & & & & & & & \\
\hline Cr 1 & 4254.336 & 0.00 & -0.114 & syn & 2.05 & 2.49 & 22.1 & 2.25 & 2.69 & & & \\
\hline Mn 1 & 4030.753 & 0.00 & -0.470 & & & 2.00 & syn & 1.95 & & & & \\
\hline Mn 1 & 4033.062 & 0.00 & -0.618 & syn & 1.50 & 2.00 & syn & 1.85 & & & & \\
\hline $\mathrm{Fe} 1$ & 3758.233 & 0.96 & -0.027 & 69.0 & 4.05 & & 78.8 & 4.37 & & & & \\
\hline $\mathrm{Fe} 1$ & 3763.789 & 0.99 & -0.238 & 51.9 & 3.80 & & 72.2 & 4.42 & & & & \\
\hline $\mathrm{Fe} 1$ & 3767.192 & 1.01 & -0.389 & 54.2 & 4.03 & & 59.7 & 4.22 & & & & \\
\hline $\mathrm{Fe} 1$ & 3786.677 & 1.01 & -2.225 & 7.6 & 4.51 & & 8.1 & 4.58 & & & & \\
\hline $\mathrm{Fe} 1$ & 3787.880 & 1.01 & -0.859 & 35.4 & 4.04 & & 45.5 & 4.31 & & & & \\
\hline $\mathrm{Fe} 1$ & 3815.840 & 1.49 & 0.237 & 61.6 & 4.03 & & 73.5 & 4.40 & & & & \\
\hline $\mathrm{Fe} 1$ & 3820.425 & 0.86 & 0.119 & 86.2 & 4.26 & & 93.3 & 4.48 & & & & \\
\hline $\mathrm{Fe} 1$ & 3824.444 & 0.00 & -1.362 & 62.1 & 4.29 & & 66.0 & 4.45 & & & & \\
\hline $\mathrm{Fe} 1$ & 3827.822 & 1.56 & 0.062 & 52.6 & 4.03 & & 54.4 & 4.11 & & & & \\
\hline $\mathrm{Fe} 1$ & 3840.437 & 0.99 & -0.506 & 54.3 & 4.12 & & 67.2 & 4.51 & & & & \\
\hline $\mathrm{Fe} 1$ & 3849.966 & 1.01 & -0.871 & 46.4 & 4.30 & & 53.6 & 4.52 & & & & \\
\hline $\mathrm{Fe} 1$ & 3850.818 & 0.99 & -1.734 & 16.7 & 4.40 & & 20.4 & 4.55 & & & & \\
\hline $\mathrm{Fe} 1$ & 3856.371 & 0.05 & -1.286 & 63.3 & 4.29 & & 67.3 & 4.46 & & & & \\
\hline $\mathrm{Fe} 1$ & 3859.911 & 0.00 & -0.710 & 75.3 & 4.04 & & 81.7 & 4.28 & & & & \\
\hline $\mathrm{Fe} 1$ & 3865.523 & 1.01 & -0.982 & 34.0 & 4.12 & & 54.6 & 4.65 & & & & \\
\hline $\mathrm{Fe} 1$ & 3878.018 & 0.96 & -0.914 & 45.8 & 4.27 & & 55.5 & 4.56 & & & & \\
\hline
\end{tabular}


Table 5. continued.

\begin{tabular}{|c|c|c|c|c|c|c|c|c|c|c|c|c|}
\hline \multirow[b]{2}{*}{ Elem } & \multirow[b]{2}{*}{$\begin{array}{c}\lambda \\
(\AA)\end{array}$} & \multirow[b]{2}{*}{$\begin{array}{r}\text { Exc. Pot. } \\
(\mathrm{eV}) \\
\end{array}$} & \multirow[b]{2}{*}{$\log g f$} & \multicolumn{3}{|c|}{ SDSS J111407+182831 } & \multicolumn{3}{|c|}{ SDSS J114323+202058 } & \multicolumn{3}{|c|}{ SDSS J220924-002859 } \\
\hline & & & & $\begin{array}{r}E W \\
(\mathrm{~m} \AA)\end{array}$ & $\begin{array}{l}\text { abund } \\
\text { LTE }\end{array}$ & $\begin{array}{l}\text { abund } \\
\text { NLTE }\end{array}$ & $\begin{array}{r}E W \\
(\mathrm{~m} \AA)\end{array}$ & $\begin{array}{r}\text { abund } \\
\text { LTE }\end{array}$ & $\begin{array}{l}\text { abund } \\
\text { NLTE }\end{array}$ & $\begin{array}{c}E W \\
(\mathrm{~m} \AA)\end{array}$ & $\begin{array}{r}\text { abund } \\
\text { LTE }\end{array}$ & $\begin{array}{l}\text { abund } \\
\text { NLTE }\end{array}$ \\
\hline $\mathrm{Fe} 1$ & 3899.707 & 0.09 & -1.531 & 55.1 & 4.32 & & 58.5 & 4.46 & & & & \\
\hline $\mathrm{Fe} 1$ & 3920.258 & 0.12 & -1.746 & 38.2 & 4.14 & & 49.0 & 4.44 & & & & \\
\hline $\mathrm{Fe} 1$ & 3922.912 & 0.05 & -1.651 & 43.7 & 4.11 & & 50.4 & 4.32 & & & & \\
\hline $\mathrm{Fe} 1$ & 4005.242 & 1.56 & -0.610 & 29.3 & 4.13 & & 33.8 & 4.27 & & & & \\
\hline Fe 1 & 4045.812 & 1.49 & 0.280 & 64.6 & 4.03 & & 69.1 & 4.19 & & 36.4 & 3.52 & \\
\hline $\mathrm{Fe} 1$ & 4063.594 & 1.56 & 0.062 & 60.8 & 4.20 & & 64.2 & 4.33 & & 31.7 & 3.69 & \\
\hline $\mathrm{Fe} 1$ & 4071.738 & 1.61 & -0.022 & 46.9 & 3.98 & & 57.7 & 4.29 & & 28.9 & 3.75 & \\
\hline $\mathrm{Fe} 1$ & 4076.629 & 3.21 & -0.529 & 10.5 & 4.97 & & & & & & & \\
\hline $\mathrm{Fe} 1$ & 4132.058 & 1.61 & -0.675 & 28.1 & 4.20 & & 24.8 & 4.15 & & & & \\
\hline $\mathrm{Fe} 1$ & 4143.868 & 1.56 & -0.511 & 35.1 & 4.15 & & 33.0 & 4.13 & & & & \\
\hline Fe 1 & 4157.780 & 3.42 & -0.403 & 26.6 & 5.54 & & 14.9 & 5.22 & & & & \\
\hline Fe 1 & 4199.095 & 3.05 & 0.155 & 44.6 & 5.07 & & 39.9 & 4.99 & & & & \\
\hline Fe 1 & 4202.029 & 1.49 & -0.708 & 24.5 & 4.02 & & 34.8 & 4.30 & & & & \\
\hline Fe 1 & 4222.213 & 2.45 & -0.967 & & & & 9.5 & 4.68 & & & & \\
\hline Fe 1 & 4227.427 & 3.33 & 0.266 & 13.9 & 4.41 & & 17.4 & 4.56 & & & & \\
\hline Fe 1 & 4250.119 & 2.47 & -0.405 & 16.1 & 4.37 & & 11.0 & 4.20 & & & & \\
\hline $\mathrm{Fe} 1$ & 4260.474 & 2.40 & 0.109 & 26.5 & 4.09 & & 26.5 & 4.11 & & & & \\
\hline Fe 1 & 4383.545 & 1.49 & 0.200 & 64.4 & 4.05 & & 67.6 & 4.18 & & 28.8 & 3.39 & \\
\hline $\mathrm{Fe} 1$ & 4404.750 & 1.56 & -0.142 & 41.9 & 3.91 & & 52.3 & 4.18 & & 17.4 & 3.49 & \\
\hline $\mathrm{Fe} 1$ & 4443.194 & 2.86 & -1.043 & 5.1 & 4.79 & & 5.2 & 4.82 & & & & \\
\hline $\mathrm{Fe} 1$ & 4466.551 & 2.83 & -0.600 & 7.4 & 4.50 & & 7.5 & 4.53 & & & & \\
\hline $\mathrm{Fe} 1$ & 4489.739 & 0.12 & -3.966 & 8.6 & 5.41 & & 4.9 & 5.19 & & & & \\
\hline $\mathrm{Fe} 1$ & 4528.614 & 2.18 & -0.822 & 10.4 & 4.28 & & 13.8 & 4.45 & & & & \\
\hline $\mathrm{Fe} 1$ & 4531.148 & 1.49 & -2.155 & 5.5 & 4.66 & & 4.3 & 4.58 & & & & \\
\hline Fe 1 & 4891.492 & 2.85 & -0.112 & 16.5 & 4.40 & & & & & & & \\
\hline $\mathrm{Fe} 1$ & 4918.994 & 2.87 & -0.342 & & & & 11.7 & 4.49 & & & & \\
\hline Fe 1 & 4920.502 & 2.83 & 0.068 & 11.3 & 4.01 & & 18.5 & 4.29 & & & & \\
\hline $\mathrm{Fe} 2$ & 4233.172 & 2.58 & -1.947 & 8.2 & 4.23 & & 7.7 & 4.20 & & & & \\
\hline Fe 2 & 4583.837 & 2.81 & -1.867 & 5.3 & 4.12 & & 8.7 & 4.37 & & & & \\
\hline $\mathrm{Fe} 2$ & 4923.927 & 2.89 & -1.320 & 16.0 & 4.18 & & 15.0 & 4.15 & & & & \\
\hline Co 1 & 3845.461 & 0.92 & 0.010 & 16.5 & 2.46 & & syn & 2.3 & & & & \\
\hline Co 1 & 3995.302 & 0.92 & -0.220 & 10.0 & 2.41 & & syn & 2.2 & & & & \\
\hline Co 1 & 4121.311 & 0.92 & -0.320 & 7.3 & 2.34 & & 6.0 & 2.29 & & & & \\
\hline Ni 1 & 3807.138 & 0.42 & -1.205 & 15.1 & 2.96 & & syn & 3.12 & & & & \\
\hline Ni 1 & 3858.292 & 0.42 & -0.936 & 23.8 & 2.95 & & syn & 2.85 & & & & \\
\hline Zn 1 & 4810.528 & 4.08 & -0.137 & 2.5 & 2.09 & & 2.7 & 2.14 & & & & \\
\hline Sr 2 & 4077.709 & 0.00 & 0.167 & 64.4 & 0.11 & 0.31 & 70.9 & 0.34 & 0.54 & $<8.0$ & $<-1.45$ & $<-1.20$ \\
\hline Sr 2 & 4215.519 & 0.00 & -0.145 & 53.5 & 0.06 & 0.26 & 59.3 & 0.26 & 0.46 & & & \\
\hline $\mathrm{Ba} 2$ & 4554.029 & 0.00 & 0.170 & syn & 0.41 & 0.41 & syn & 0.95 & 0.95 & $<10.0$ & $<-1.04$ & $<-1.04$ \\
\hline Ba 2 & 5853.668 & 0.60 & -1.000 & & & & syn & 0.80 & 0.80 & & & \\
\hline Ba 2 & 6141.713 & 0.70 & -0.076 & 36.6 & 0.42 & 0.42 & 52.5 & 0.85 & 0.85 & & & \\
\hline Ba 2 & 6496.897 & 0.60 & -0.377 & 32.0 & 0.50 & 0.50 & 44.6 & 0.83 & 0.83 & & & \\
\hline
\end{tabular}

